\begin{tabular}{|c|c|}
\hline Citation & $\begin{array}{l}\text { Florian Maurin, Francesco Greco, Laurens Coox, Dirk Vandepitte, Wim } \\
\text { Desmet, (2018), } \\
\text { Title: Isogeometric collocation for Kirchhoff-Love plates and shells } \\
\text { Computer Methods in Applied Mechanics and Engineering, Volume 329, } \\
\text { Pages 396-420 }\end{array}$ \\
\hline Archived version & $\begin{array}{l}\text { Author manuscript: the content is identical to the content of the published } \\
\text { paper, but without the final typesetting by the publisher }\end{array}$ \\
\hline Published version & 10.1016/j.cma.2017.10.007 \\
\hline Journal homepage & http://www.sciencedirect.com/science/article/pii/S004578251730676X \\
\hline Author contact & $\begin{array}{l}\text { florian.maurin@kuleuven.be } \\
+32 \text { (0)16 } 372391\end{array}$ \\
\hline IR & https://lirias.kuleuven.be/handle/123456789/597968 \\
\hline
\end{tabular}

(article begins on next page) 


\title{
Isogeometric collocation for Kirchhoff-Love plates and shells
}

\author{
Florian Maurin ${ }^{\mathrm{a}, \mathrm{b}, *}$, Francesco Greco ${ }^{\mathrm{a}, \mathrm{b}}$, Laurens Coox $^{\mathrm{a}, \mathrm{b}}$, Dirk Vandepitte ${ }^{\mathrm{a}, \mathrm{b}}$, \\ Wim Desmet ${ }^{\mathrm{a}, \mathrm{b}}$ \\ ${ }^{a} K U$ Leuven, Department of Mechanical Engineering, Division PMA \\ Celestijnenlaan 300B, B-3001 Leuven, Belgium. \\ ${ }^{b}$ Member of Flanders Make.
}

\begin{abstract}
With the emergence of isogeometric analysis (IGA), the Galerkin rotation-free discretization of Kirchhoff-Love shells is facilitated, enabling more efficient thin shell structural analysis. High-order shape functions used in IGA also allow the collocation of partial differential equations, avoiding the time-consuming numerical integration of the Galerkin technique. The goal of the present work is to apply this method to NURBS-based isogeometric Kirchhoff-Love plates and shells, under the assumption of small deformations.

Since Kirchhoff-Love plate theory yields a fourth-order formulation, two boundary conditions are required at each location on the contour, generating some conflicts at the corners where there are more equations than needed. To remedy this overdetermination, we provide priority and averaging rules that cover all the possible combinations of adjacent edge boundary conditions (i.e. the clamped, simplysupported, symmetric and free supports). Greville and alternative superconvergent points are used for NURBS basis of even and odd degrees, respectively. For square, circular, and annular flat plates, convergence orders are found to be in agreement with a-priori error estimates. The proposed isogeometric collocation method is then validated and benchmarked against a Galerkin implementation by studying a set of problems involving Kirchhoff-Love shells.
\end{abstract}

Keywords: Isogeometric collocation, Kirchhoff-Love shell, rotation-free discretization, convergence order

*Corresponding author: florian.maurin@kuleuven.be 


\section{Introduction}

First introduced by Hughes et al. [1, 2], isogeometric analysis (IGA) aims at bridging the gap between computer aided design (CAD) and finite element analysis (FEA), by considering an isoparametric approach for which the basis functions used for the geometry representations in CAD systems are also used to approximate the field variables. These shape functions are usually non-uniform rational B-splines (NURBS) and their continuity can be augmented through the $k$ refinement procedure, improving the accuracy and robustness of the simulations [2]. High-order continuous shape functions allow also direct solutions to highorder partial differential equations (PDEs) such as the incompressible elasticity with stream functions [3], the Cahn-Hilliard phase-field model [4], the LaplaceBeltrami triharmonic problem [5], and the Navier-Stokes equations [6]. In structural mechanics, rotation-free formulations can be obtained for beams [7-9] as well as for shells, the focus of the present manuscript.

Starting from the pioneering works of Kiendl et al. [10], isogeometric KirchhoffLove shell formulations have been extended to non-linear material models [11, 12], analysis of through-the-thickness cracks [13], damage models [14], and biological membranes [15]. Due to the rotation-free nature of these formulations, multi-patch coupling deserves special attention [12, 16-20].

High-order continuous shape functions allow also the collocation of the strong form, leading to the field of isogeometric collocation methods initially investigated by Auricchio et al. [21]. A cost comparison with the Galerkin method can be found in [22]. In [23, 24], a hybrid collocation-Galerkin method is introduced to weakly enforce Neumann conditions, avoiding spurious oscillations of the purely collocated solutions. Isogeometric collocation has been applied to elastostatics and explicit dynamics [25], phase-field models [26, 27], contact problems [23, 24, 28], Timoshenko beams [29-34], Reissner-Mindlin plates and shells [35-37] and Kirchhoff-Love plates [38, 39]. It is the goal of the present paper to extend the work of [38] to Kirchhoff-Love shells.

Greville abscissae (C-GP) are often chosen as collocation points, and for secondorder PDEs, the $L^{2}$-error decays as $\mathcal{O}\left(h^{p}\right)$ and $\mathcal{O}\left(h^{p-1}\right)$ for even and odd NURBS degree $p$, respectively, whereas the Galerkin method converges at an optimal order of $\mathcal{O}\left(h^{p+1}\right)$ ( $h$ being the mesh size). It has been recently shown that for uniform meshes, the optimal convergence order with the collocation method can be obtained by using Cauchy-Galerkin points also called superconvergent points [4042]. However, the number of these points is about two times higher than the number of control points for a univariate spline. This overdetermined system can 
be solved using the least-squares approximation at superconvergent points (LSSP) [40]. Another approach, as proposed in [42], is to select a subset formed by every other point; these points are referred to as alternating superconvergent points (C-ASP). However, for odd degrees, the $L^{2}$-error decays as $\mathcal{O}\left(h^{p+1}\right)$ with the use of LS-SP, whereas C-ASP provide a convergence order of only $\mathcal{O}\left(h^{p}\right)$. It is shown in [42] that a more appropriate subset can be defined based on element symmetries, and this method is referred to as clustered superconvergent points (C-CSP). While the use of LS-SP or C-CSP provides an optimal convergence for odd degrees, the convergence for even degrees is suboptimal and remains an open challenge [42]. Because C-CSP are only given for second-order problems in [42], the present manuscript uses C-ASP instead; their definition for fourth-order problems is detailed in [41].

The paper is organized as follows. In Section 2, the Kirchhoff-Love shell theory is summarized. In Section 3, after a short introduction to NURBS-based IGA, the Galerkin discretization of the strong and weak forms are presented, followed by the different strategies to collocate the strong form. Section 4 then illustrates a number of numerical case studies, allowing a comparison between the Galerkin and the collocation methods.

\section{Kirchhoff-Love shell}

Before introducing the Kirchhoff-Love shell theory, differential geometry of surfaces is summarized based on [43].

\subsection{Differential geometry of surfaces}

In the following, Einstein's summation convention is used and indices in Greek and Latin letters take the values $\{1,2\}$ and $\{1,2,3\}$, respectively. Assuming a thin shell, its mid-surface can be described by a general mapping

$$
\boldsymbol{x}=\boldsymbol{x}\left(\theta^{1}, \theta^{2}\right),
$$

where $\theta^{1}$ and $\theta^{2}$ are the convective curvilinear coordinates of the surface. With the covariant base vectors

$$
\boldsymbol{a}_{\alpha}=\boldsymbol{x},_{\alpha}=\frac{\partial \boldsymbol{x}}{\partial \theta^{\alpha}},
$$

the covariant metric coefficients of the surface (first fundamental form of the surface) are defined as

$$
a_{\alpha \beta}=a_{\beta \alpha}=\boldsymbol{a}_{\alpha} \cdot \boldsymbol{a}_{\beta},
$$


and the contravariant base vectors are obtained by

$$
\boldsymbol{a}^{\alpha}=a^{\alpha \beta} \boldsymbol{a}_{\beta} \quad \text { with } \quad\left[a^{\alpha \beta}\right]=\left[a_{\alpha \beta}\right]^{-1},
$$

where $\left[a_{\alpha \beta}\right]$ is the $2 \times 2$ matrix formed by the coefficients $a_{\alpha \beta}$. The curvature tensor coefficients are defined by the second fundamental form of the surface

$$
b_{\alpha \beta}=b_{\beta \alpha}=\boldsymbol{a}_{\alpha, \beta} \cdot \boldsymbol{a}_{3},
$$

where $\boldsymbol{a}_{3}=\boldsymbol{a}^{3}$ is the unit normal vector of the mid-surface:

$$
\boldsymbol{a}_{3}=\frac{\widetilde{\boldsymbol{a}}_{3}}{\bar{a}_{3}} \quad \text { with } \quad \widetilde{\boldsymbol{a}}_{3}=\boldsymbol{a}_{1} \times \boldsymbol{a}_{2} \quad \text { and } \quad \bar{a}_{3}=\sqrt{\widetilde{\boldsymbol{a}}_{3} \cdot \widetilde{\boldsymbol{a}}_{3}} .
$$

The parametric derivatives of $\boldsymbol{a}_{\alpha}$ are expressed in the covariant basis as

$$
\boldsymbol{a}_{\alpha, \beta}=\boldsymbol{a}_{\beta, \alpha}=\frac{\partial \boldsymbol{a}_{\alpha}}{\partial \theta^{\beta}}=\Gamma_{\alpha \beta}^{\rho} \boldsymbol{a}_{\rho}+\Gamma_{\alpha \beta}^{3} \boldsymbol{a}_{3},
$$

where the Christoffel symbol $\Gamma_{\alpha \beta}^{\rho}$ is defined by

$$
\Gamma_{\alpha \beta}^{\rho}=\boldsymbol{a}_{\alpha, \beta} \cdot \boldsymbol{a}^{\rho} \quad \text { and } \quad \Gamma_{\alpha \beta}^{3}=\boldsymbol{a}_{\alpha, \beta} \cdot \boldsymbol{a}_{3} .
$$

The curvature and metric tensor coefficients are linked to the non-symmetric tensor coefficients $b_{\beta}^{\alpha}$ such that

$$
b^{\alpha \beta}=b_{\rho}^{\alpha} a^{\rho \beta} \quad \text { with } \quad b_{\beta}^{\alpha}=b_{\beta \rho} a^{\rho \alpha} .
$$

The mean and Gaussian curvatures are defined from the first and second fundamental forms of the surface, respectively, as

$$
H=\frac{1}{2} b_{\alpha}^{\alpha}=\frac{1}{2} a^{\alpha \beta} b_{\alpha \beta},
$$

and

$$
K=\frac{\operatorname{det}\left[b_{\alpha \beta}\right]}{\operatorname{det}\left[a_{\alpha \beta}\right]} .
$$

Note that for a flat surface, $b_{\alpha}^{\beta}=\Gamma_{\alpha \beta}^{\rho}=0$, whereas for a uniformly curved one (e.g. a cylinder or a sphere), $\left(b_{\alpha}^{\beta}\right)_{, \alpha}=\left(b_{\alpha}^{\beta}\right)_{, \beta}=0$. 


\subsection{Kirchhoff-Love shell theory}

In the Kirchhoff-Love theory, the shell thickness $t=t\left(\theta^{1}, \theta^{2}\right)$ is assumed to be small such that transverse shear strains have only a negligible contribution to elastic energy which is stored in the deformed shell. Consequently, a vector normal to the mid-surface remains normal in the actual configuration and the shell parametrization is reduced to its mid-surface. Displacement of the shell midsurface at the position $\left(\theta^{1}, \theta^{2}\right)$ reads [43]

$$
\boldsymbol{u}\left(\theta^{1}, \theta^{2}\right)=\boldsymbol{x}\left(\theta^{1}, \theta^{2}\right)-\boldsymbol{X}\left(\theta^{1}, \theta^{2}\right),
$$

where $\boldsymbol{X}$ and $\boldsymbol{x}$ are the position vectors in the reference and the actual configuration, respectively. Whereas $\boldsymbol{a}_{\alpha}, \boldsymbol{a}^{3}, a_{\alpha \beta}$ and $b_{\alpha \beta}$ are derived in the actual configuration, equivalent quantities in the reference configuration are derived similarly and are denoted respectively $\boldsymbol{A}_{\alpha}, \boldsymbol{A}^{3}, A_{\alpha \beta}$ and $B_{\alpha \beta}$.

The components of the Green-Lagrange strain tensor are split into membrane and bending actions as follows:

$$
E_{(\alpha \beta)}=\epsilon_{(\alpha \beta)}-\theta^{3} \kappa_{(\alpha \beta)},
$$

where $\theta^{3} \in[-t / 2, t / 2]$ is the thickness coordinate. The membrane strain and the curvature change are respectively defined by

$$
\begin{aligned}
\epsilon_{(\alpha \beta)} & =\frac{1}{2}\left(a_{\alpha \beta}-A_{\alpha \beta}\right), \\
\kappa_{(\alpha \beta)} & =-\left(b_{\alpha \beta}-B_{\alpha \beta}\right) .
\end{aligned}
$$

The linear Hooke's law is employed for the description of the constitutive behavior of the elastic material and reads

$$
\bar{S}^{(\alpha \beta)}=\mathrm{C}^{\alpha \beta \gamma \delta} \bar{E}_{(\gamma \delta)},
$$

where $\mathrm{C}^{\alpha \beta \gamma \delta}$ is the fourth-order elastic tensor and $\boldsymbol{\bullet}$ indicates that the quantities are expressed in a local Cartesian system. The symbol $\bullet \bullet$ or $\bullet(\bullet)$ indicates that the tensor is symmetric (i.e. $\bullet_{(\alpha \beta)}=\bullet_{(\beta \alpha)}$ ). Using the fact that both $\bar{S}^{(\alpha \beta)}$ and $\bar{E}_{(\gamma \delta)}$ are symmetric, and considering an isotropic material with Young's modulus $E$ and Poisson ratio $\nu$, Eq. (15) is simplified using Voigt notation as:

$$
\left[\begin{array}{c}
\bar{S}^{(11)} \\
\bar{S}^{(22)} \\
\bar{S}^{(12)}
\end{array}\right]=\overline{\mathrm{D}}\left[\begin{array}{c}
\bar{E}_{(11)} \\
\bar{E}_{(22)} \\
2 \bar{E}_{(12)}
\end{array}\right], \quad \text { with } \quad \overline{\mathrm{D}}=\frac{E}{1-\nu^{2}}\left[\begin{array}{ccc}
1 & \nu & 0 \\
\nu & 1 & 0 \\
0 & 0 & \frac{1-\nu}{2}
\end{array}\right] \text {. }
$$


For more complex materials described by orthotropic or nonlinear stress-strain relations, we refer the reader to $[11,12]$. Expressing the local orthonormal basis $\overline{\boldsymbol{E}}_{\alpha}$ in the undeformed configuration in terms of the covariant basis $\overline{\boldsymbol{A}}_{\alpha}$ such that

$$
\begin{aligned}
& \overline{\boldsymbol{E}}_{1}=\frac{\boldsymbol{A}_{1}}{\left\|\boldsymbol{A}_{1}\right\|}, \\
& \overline{\boldsymbol{E}}_{2}=\frac{\boldsymbol{A}_{2}-\left(\boldsymbol{A}_{2} \cdot \overline{\boldsymbol{E}}_{1}\right) \overline{\boldsymbol{E}}_{1}}{\left\|\boldsymbol{A}_{2}-\left(\boldsymbol{A}_{2} \cdot \overline{\boldsymbol{E}}_{1}\right) \overline{\boldsymbol{E}}_{1}\right\|},
\end{aligned}
$$

the stress and strain tensors in the contravariant and covariant basis are respectively linked by

$$
\begin{aligned}
& \bar{E}_{(\gamma \delta)}=E_{\alpha \beta}\left(\overline{\boldsymbol{E}}_{\gamma} \cdot \boldsymbol{A}^{\alpha}\right)\left(\overline{\boldsymbol{E}}_{\delta} \cdot \boldsymbol{A}^{\beta}\right), \\
& \bar{S}^{(\gamma \delta)}=S^{\alpha \beta}\left(\overline{\boldsymbol{E}}_{\gamma} \cdot \boldsymbol{A}_{\alpha}\right)\left(\overline{\boldsymbol{E}}_{\delta} \cdot \boldsymbol{A}_{\beta}\right) .
\end{aligned}
$$

Consequently, the strain-stress relation reads:

$$
\left[\begin{array}{l}
S^{(11)} \\
S^{(22)} \\
S^{(12)}
\end{array}\right]=\mathrm{D}\left[\begin{array}{c}
E_{(11)} \\
E_{(22)} \\
2 E_{(12)}
\end{array}\right],
$$

with

$$
\begin{aligned}
\mathrm{D}= & \frac{E}{1-\nu^{2}} \frac{1}{\left(A_{11} A_{22}-A_{12}^{2}\right)^{2}} \\
& {\left[\begin{array}{ccc}
A_{22}^{2} & (1-\nu) A_{12}^{2}+\nu A_{11} A_{22} & -A_{22} A_{12} \\
(1-\nu) A_{12}^{2}+\nu A_{11} A_{22} & A_{11}^{2} & -A_{11} A_{12} \\
-A_{22} A_{12} & -A_{11} A_{12} & \frac{1}{2}\left((1+\nu) A_{12}^{2}+(1-\nu) A_{11} A_{22}\right)
\end{array}\right] . }
\end{aligned}
$$

After integration over the thickness, the stress resultants $n^{(\alpha \beta)}$ for normal forces and $m^{(\alpha \beta)}$ for bending moments read:

$$
\begin{aligned}
n^{(\alpha \beta)} & =t \mathrm{C}^{\alpha \beta \gamma \delta} \epsilon_{(\gamma \delta)}, \\
m^{(\alpha \beta)} & =\frac{t^{3}}{12} \mathrm{C}^{\alpha \beta \gamma \delta} \kappa_{(\gamma \delta)} .
\end{aligned}
$$

\subsection{Boundary value problem}

Consider a shell that is $G^{1}$-continuous inside its domain $\Omega$, whereas its contour $\Gamma$ can be non-smooth (e.g. corners), as illustrated in Fig. 1. Let $\tau$ be the unit vector tangent to $\Gamma$ and $\nu$ the outward unit vector normal to $\Gamma$, and tangent to $\Omega$ such that

$$
\nu=\tau \times a_{3} .
$$




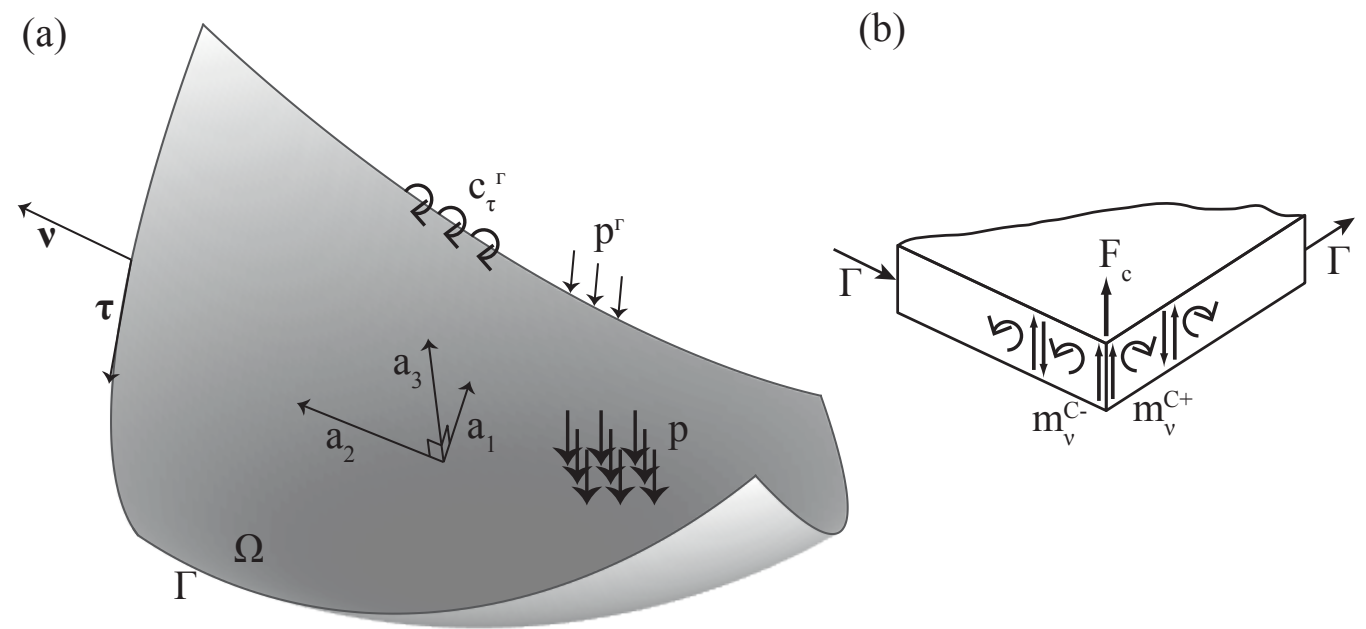

Figure 1: The different prescribed actions applied to a shell (a), and details on the shear corner force $F_{c}$ induced by the moment normal to the contour $m_{\nu}$ (b).

From equilibrium conditions, the Kirchhoff-Love shell boundary value problem (BVP) reads [43]

$$
\begin{aligned}
\left.n^{\alpha \beta}\right|_{\alpha}-q^{\alpha} b_{\alpha}^{\beta}+p^{\beta} & =0 \quad \text { in } \Omega, \\
n^{\alpha \beta} b_{\alpha \beta}+\left.q^{\alpha}\right|_{\alpha}+p^{3} & =0 \text { in } \Omega, \\
\boldsymbol{u} & =\boldsymbol{u}^{\Gamma} \text { on } \Gamma, \\
\omega_{\tau} & =\omega_{\tau}^{\Gamma} \text { on } \Gamma, \\
\boldsymbol{n} & =\boldsymbol{p}^{\Gamma} \text { on } \Gamma, \\
m_{\tau} & =c_{\tau}^{\Gamma} \text { on } \Gamma,
\end{aligned}
$$

where $\boldsymbol{q}=q^{\alpha} \boldsymbol{a}_{\alpha}$ is the shear force defined by

$$
q^{\beta}=\left.m^{\alpha \beta}\right|_{\alpha},
$$

and

$$
\begin{aligned}
\left.n^{\alpha \beta}\right|_{\alpha} & =n_{, \alpha}^{\alpha \beta}+n^{\rho \beta} \Gamma_{\alpha \rho}^{\alpha}+n^{\alpha \rho} \Gamma_{\alpha \rho}^{\beta}, \\
\left.m^{\alpha \beta}\right|_{\alpha} & =m_{, \alpha}^{\alpha \beta}+m^{\rho \beta} \Gamma_{\alpha \rho}^{\alpha}+m^{\alpha \rho} \Gamma_{\alpha \rho}^{\beta}, \\
\left.q^{\alpha}\right|_{\alpha} & =q_{, \alpha}^{\alpha}+q^{\rho} \Gamma_{\alpha \rho}^{\alpha} .
\end{aligned}
$$


Eq. (23a) and (23b) describe equilibrium of forces in the direction $\boldsymbol{a}_{\beta}$ and $\boldsymbol{a}_{3}$, respectively. Contrary to $m^{\alpha \beta}, n^{\alpha \beta}$ is in general not symmetric and it is given by

$$
\begin{aligned}
& n^{(\alpha \beta)}=n^{\alpha \beta}+m^{\alpha \rho} b_{\rho}^{\beta}, \\
& m^{(\alpha \beta)}=m^{\alpha \beta} .
\end{aligned}
$$

Inside the domain $\Omega, \boldsymbol{p}=p^{\alpha} \boldsymbol{a}_{\alpha}+p^{3} \boldsymbol{a}_{3}$ is the prescribed body force. At the contour $\Gamma$, Eqs. (23c) and (23d) are Dirichlet boundary conditions enforcing the displacement $\boldsymbol{u}^{\Gamma}$ and the rotation $\omega_{\tau}^{\Gamma}$ around the edge $\tau$, whereas Eqs. (23e) and (23f) represent Neumann boundary conditions with the prescribed force $p^{\Gamma}$ and moment (bending) $\boldsymbol{c}^{\Gamma}=c_{\tau}^{\Gamma} \boldsymbol{\tau}$ which is assumed to be purely around $\boldsymbol{\tau}$. The displacement, rotation, force and moment at the contour are decomposed into

$$
\begin{aligned}
\boldsymbol{u} & =u_{\tau} \boldsymbol{\tau}+u_{\nu} \boldsymbol{\nu}+u_{3} \boldsymbol{a}_{3}, & \boldsymbol{n} & =n_{\tau} \boldsymbol{\tau}+n_{\nu} \boldsymbol{\nu}+n_{3} \boldsymbol{a}_{3}, \\
\boldsymbol{\omega} & =\omega_{\tau} \boldsymbol{\tau}+\omega_{\nu} \boldsymbol{\nu}, & \boldsymbol{m} & =m_{\tau} \boldsymbol{\tau}+m_{\nu} \boldsymbol{\nu},
\end{aligned}
$$

where

$$
\begin{aligned}
u_{\tau} & =\tau^{\alpha} u_{\alpha}=\boldsymbol{\tau} \cdot \boldsymbol{u} \\
u_{\nu} & =\nu^{\alpha} u_{\alpha}=\boldsymbol{\nu} \cdot \boldsymbol{u} \\
\omega_{\tau} & =-\frac{d \boldsymbol{u}_{3}}{d \nu}-\nu^{\beta} b_{\beta}^{\lambda} \boldsymbol{u}_{\lambda}=-\nu^{\beta} \boldsymbol{u}_{3, \beta}-\nu^{\beta} b_{\beta}^{\lambda} \boldsymbol{u}_{\lambda} \\
& =-\nu^{\beta}\left(\boldsymbol{a}_{3, \beta}+b_{\beta}^{\lambda} \boldsymbol{a}_{\lambda}\right) \cdot \boldsymbol{u}-\nu^{\beta} \boldsymbol{a}_{3} \cdot \boldsymbol{u}_{, \beta},
\end{aligned}
$$

and

$$
\begin{aligned}
& n_{\tau}=n^{\alpha \beta} \nu_{\alpha} \tau_{\beta}=n_{\tau}\left(\epsilon_{(\alpha \beta)}, \kappa_{(\alpha \beta)}\right), \\
& n_{\nu}=n^{\alpha \beta} \nu_{\alpha} \nu_{\beta}=n_{\nu}\left(\epsilon_{(\alpha \beta)}, \kappa_{(\alpha \beta)}\right), \\
& n_{3}=q^{\alpha} \nu_{\alpha}=n_{3}\left(\kappa_{(\alpha \beta)}, \kappa_{(\alpha \beta), \gamma}\right), \\
& m_{\tau}=m^{\alpha \beta} \nu_{\alpha} \nu_{\beta}=m_{\tau}\left(\kappa_{(\alpha \beta)}\right), \\
& m_{\nu}=-m^{\alpha \beta} \nu_{\alpha} \tau_{\beta}=m_{\nu}\left(\kappa_{(\alpha \beta)}\right) .
\end{aligned}
$$

\subsection{Weak form}

In order to validate the implementation of the different terms, the Galerkin method applied to the weak form of the fourth and second-order problems is detailed in the following, and the numerical equality between the resulting stiffness matrices is checked in Section 4. 
As shown in Eq. (29), five boundary conditions have to be enforced which is not compatible with the BVP order (Eq. (23)). To reduce the number of boundary equations, integration by parts is used to convert the torsional component of the moment $\left(m_{\nu}\right)$ into a shear force term $[43,44]$. The weak form of the BVP at the contour then reads:

$$
\begin{aligned}
\delta W_{\Gamma}= & \oint_{\Gamma}\left(\left(\boldsymbol{n}-\boldsymbol{p}^{\Gamma}\right) \cdot \delta \boldsymbol{v}+\left(\boldsymbol{m}-\boldsymbol{c}^{\Gamma}\right) \cdot \delta \boldsymbol{\omega}\right) d \Gamma \\
= & \oint_{\Gamma}\left(n_{\tau} \delta v_{\tau}+n_{\nu} \delta v_{\nu}+n_{3} \delta v_{3}+m_{\tau} \delta \omega_{\tau}+m_{\nu} \delta \omega_{\nu}\right) d \Gamma \\
& -\oint_{\Gamma}\left(p_{\tau}^{\Gamma} \delta v_{\tau}+p_{\nu}^{\Gamma} \delta v_{\nu}+p_{3}^{\Gamma} \delta v_{3}+c_{\tau}^{\Gamma} \delta \omega_{\tau}\right) d \Gamma \\
= & \oint_{\Gamma}\left(\widetilde{n}_{\tau} \delta v_{\tau}+\widetilde{n}_{\nu} \delta v_{\nu}+\widetilde{n}_{3} \delta v_{3}+m_{\tau} \delta \omega_{\tau}\right) d \Gamma+\left[m_{\nu} \delta v_{3}\right]_{\mathrm{C}^{-}}^{\mathrm{C}^{+}} \\
& -\oint_{\Gamma}\left(p_{\tau}^{\Gamma} \delta v_{\tau}+p_{\nu}^{\Gamma} \delta v_{\nu}+p_{3}^{\Gamma} \delta v_{3}+c_{\tau}^{\Gamma} \delta \omega_{\tau}\right) d \Gamma,
\end{aligned}
$$

where $\delta$ is the variational symbol, $\boldsymbol{v}$ a test function, $\boldsymbol{\omega}=\boldsymbol{\omega}(\boldsymbol{v})$, and

$$
\begin{aligned}
& \widetilde{n}_{\tau}=n_{\tau}+b_{\beta}^{\lambda} t^{\beta} \tau_{\lambda} m_{\nu}=n_{\tau}+\widehat{n}_{\tau}=\widetilde{n}_{\tau}\left(\epsilon_{(\alpha \beta)}, \kappa_{(\alpha \beta)}\right), \\
& \widetilde{n}_{\nu}=n_{\nu}+b_{\beta}^{\lambda} t^{\beta} \nu_{\lambda} m_{\nu}=n_{\nu}+\widehat{n}_{\nu}=\widetilde{n}_{\nu}\left(\epsilon_{(\alpha \beta)}, \kappa_{(\alpha \beta)}\right), \\
& \tilde{n}_{3}=n_{3}-m_{\nu, \alpha} t^{\alpha}=n_{3}+\widehat{n}_{3}=\widetilde{n}_{3}\left(\kappa_{(\alpha \beta)}, \kappa_{(\alpha \beta), \gamma}\right) .
\end{aligned}
$$

The punctual shear forces $F_{C}=\left[m_{\nu} u_{3}\right]_{\mathrm{C}^{-}}^{\mathrm{C}^{+}}$are located at the contour discontinuities (e.g. corners), as illustrated in Fig. 1b, and are specific to Kirchhoff-Love theory $[43,44]$.

At the domain interior, the weak form of Eqs. (23a) and (23b) reads:

$$
\delta W_{\Omega}=-\int_{\Omega}\left(\mathcal{N}^{\beta} \boldsymbol{a}_{\beta}+\mathcal{M} \boldsymbol{a}_{3}\right) \delta \boldsymbol{v} d \Omega-\int_{\Omega}\left(p^{\beta} \boldsymbol{a}_{\beta}+p^{3} \boldsymbol{a}_{3}\right) \delta \boldsymbol{v} d \Omega=0
$$

where

$$
\begin{aligned}
& \mathcal{N}^{\beta}=\left.n^{\alpha \beta}\right|_{\alpha}-\left.m^{\alpha \beta}\right|_{\beta} b_{\alpha}^{\beta}=\mathcal{N}^{\beta}\left(\epsilon_{(\alpha \beta)}, \epsilon_{(\alpha \beta), \gamma}, \kappa_{(\alpha \beta)}, \kappa_{(\alpha \beta), \gamma}\right) \\
& \mathcal{M}=n^{\alpha \beta} b_{\alpha \beta}+\left.\left(\left.m^{\alpha \beta}\right|_{\beta}\right)\right|_{\alpha}=\mathcal{M}\left(\epsilon_{(\alpha \beta)}, \epsilon_{(\alpha \beta), \gamma}, \kappa_{(\alpha \beta)}, \kappa_{(\alpha \beta), \gamma}, \kappa_{(\alpha \beta), \gamma, \zeta}\right) .
\end{aligned}
$$

The total virtual work of the shell is expressed as the sum of Eqs. (30) and 
(32):

$$
\begin{aligned}
\delta W= & -\int_{\Omega}\left(\mathcal{N}^{\beta} \boldsymbol{a}_{\beta}+\mathcal{M} \boldsymbol{a}_{3}\right) \delta \boldsymbol{v} d \Omega-\int_{\Omega}\left(p^{\beta} \boldsymbol{a}_{\beta}+p^{3} \boldsymbol{a}_{3}\right) \delta \boldsymbol{v} d \Omega \\
& +\oint_{\Gamma}\left(\widetilde{n}_{\tau} \delta v_{\tau}+\widetilde{n}_{\nu} \delta v_{\nu}+\widetilde{n}_{3} \delta v_{3}+m_{\tau} \delta \omega_{\tau}\right) d \Gamma+\left[m_{\nu} \delta v_{3}\right]_{\mathrm{C}^{-}}^{\mathrm{C}^{+}} \\
& -\oint_{\Gamma}\left(p_{\tau}^{\Gamma} \delta v_{\tau}+p_{\nu}^{\Gamma} \delta v_{\nu}+p_{3}^{\Gamma} \delta v_{3}+c_{\tau}^{\Gamma} \delta \omega_{\tau}\right) d \Gamma=0 .
\end{aligned}
$$

This is a fourth-order equation that can be reduced to a second-order one [43]:

$$
\begin{aligned}
\delta W= & \int_{\Omega}\left(\widetilde{n}^{(\alpha \beta)} \delta \epsilon_{(\alpha \beta)}+\widetilde{m}^{(\alpha \beta)} \delta \kappa_{(\alpha \beta)}\right) d \Omega-\int_{\Omega}\left(p^{\beta} \boldsymbol{a}_{\beta}+p^{3} \boldsymbol{a}_{3}\right) \delta \boldsymbol{v} d \Omega \\
& -\oint_{\Gamma}\left(p_{\tau}^{\Gamma} \delta v_{\tau}+p_{\nu}^{\Gamma} \delta v_{\nu}+p_{3}^{\Gamma} \delta v_{3}+c_{\tau}^{\Gamma} \delta \omega_{\tau}\right) d \Gamma=0 .
\end{aligned}
$$

As shown next, the Galerkin discretization of Kirchhoff-Love shells is most of the time based on this last equation. Before going into this, the following section first introduces some preliminaries on NURBS-based IGA.

\section{Numerical discretization}

\subsection{NURBS-based IGA}

The geometry is represented by means of NURBS [2] and only single patches are considered here. A B-spline of polynomial degree $p$ is defined by a knot vector $\Xi=\left\{\xi_{1} \leq \ldots \leq \xi_{n+p+1}\right\}$, with $n$ the number of basis functions forming the Bspline. The $i^{\text {th }}(i=1, \ldots, n) \mathrm{B}$-spline basis function $N_{i}^{p}(\xi)$ is derived from the knot-vector using the Cox-De Boor recursive formula [45]:

$$
\begin{aligned}
& N_{i}^{0}(\xi)= \begin{cases}1 & \text { if } \quad \xi_{i} \leq \xi<\xi_{i+1}, \\
0 & \text { otherwise },\end{cases} \\
& N_{i}^{p}(\xi)=\frac{\xi-\xi_{i}}{\xi_{i+p}-\xi_{i}} N_{i}^{p-1}(\xi)+\frac{\xi_{i+p+1}-\xi}{\xi_{i+p+1}-\xi_{i+1}} N_{i+1}^{p-1}(\xi), \quad \text { for } p \geq 1 .
\end{aligned}
$$

In the following, for the sake of clarity, the knot vectors are open (or clamped), i.e. the multiplicity of the first and last knot is $p+1$, whereas the multiplicity of the remaining knots is 1 , such that the basis functions are $\mathcal{C}^{p-1}$-continuous. Given two univariate B-spline bases $N_{i}^{p}(\xi)(i=1, \ldots, n)$ and $M_{j}^{q}(\eta)(j=1, \ldots, m)$, respectively of order $p$ and $q$, and associated to the two knot vectors $\Xi=\left\{\xi_{1} \leq\right.$ 
$\left.\ldots \leq \xi_{n+p+1}\right\}$ and $\mathcal{H}=\left\{\eta_{1} \leq \ldots \leq \eta_{m+q+1}\right\}$, a bivariate NURBS basis function is defined as

$$
R_{i, j}^{p, q}(\xi, \eta)=\frac{N_{i}^{p}(\xi) M_{j}^{q}(\eta) w_{i, j}}{\sum_{i_{w}=1}^{n} \sum_{j_{w}=1}^{m} N_{i_{w}}^{p}(\xi) M_{j_{w}}^{q}(\eta) w_{i_{w}, j_{w}}},
$$

where $w_{i, j} \in \mathbb{R}$ is the weight associated to the basis function $R_{i, j}^{p, q}$. NURBS surfaces $\boldsymbol{S}(\xi, \eta)$ are defined by

$$
\boldsymbol{S}(\xi, \eta)=\sum_{i=1}^{n} \sum_{j=1}^{m} R_{i, j}^{p, q}(\xi, \eta) \mathbf{B}_{i, j}=\sum_{r=1}^{n \cdot m} R_{r}^{p, q}(\xi, \eta) \mathbf{B}_{r}
$$

where $\mathbf{B}_{i, j}$ are the control points and with $r=i+n(j-1)$ a single counter replacing $i$ and $j$. Using the isogeometric approach, the displacement field $\boldsymbol{u}(\xi, \eta)$ is approximated by the same basis functions that represent the geometry such that

$$
\boldsymbol{u}(\xi, \eta)=\sum_{r=1}^{n \cdot m} R_{r}^{p, q}(\xi, \eta) \mathbf{u}_{r}
$$

where $\mathbf{u}_{r}$ is the global displacement control variable corresponding to the control point $\mathbf{B}_{r}$.

\subsection{Galerkin method}

The Bubnov-Galerkin approach considers as test functions the same shape functions that describe the solution field:

$$
\boldsymbol{v}(\xi, \eta)=\sum_{r=1}^{n \cdot m} R_{r}^{p, q}(\xi, \eta) \mathbf{v}_{r}
$$

In the present work, for the sake of simplicity, the deformations are assumed to be small, such that the geometric stiffness is neglected in the linearization of Eqs. (34) and (35). The resulting stiffness matrix components for the fourth-order problem (34) read

$$
\begin{aligned}
\mathrm{K}_{r, s}^{\left(G_{4}\right)}= & \sum_{e=1}^{n_{e}}\left[-\int_{\Omega_{e}} v_{r}\left(\boldsymbol{a}_{\beta} \mathcal{N}_{s}^{\beta^{T}}+\boldsymbol{a}_{3} \mathcal{M}^{T}\right) d \Omega\right. \\
& +\oint_{\Gamma_{e}} v_{r}\left(\boldsymbol{\tau} \widetilde{\boldsymbol{n}}_{\tau, s}^{T}+\boldsymbol{\nu} \widetilde{\boldsymbol{n}}_{\nu, s}^{T}+\boldsymbol{a}_{3} \widetilde{\boldsymbol{n}}_{3, s}^{T}-\nu^{\beta}\left(\boldsymbol{a}_{3, \beta}+b_{\beta}^{\lambda} \boldsymbol{a}_{\lambda}\right) \boldsymbol{m}_{\tau, s}^{T}\right) d \Gamma \\
& \left.-\oint_{\Gamma_{e}} v_{\beta, r} \nu^{\beta} \boldsymbol{a}_{3} \boldsymbol{m}_{\tau, s}^{T} d \Gamma\right]+\left[v_{r} \boldsymbol{a}_{3} \boldsymbol{m}_{\nu}^{T}\right]_{\mathrm{C}^{-}}^{\mathrm{C}^{+}},
\end{aligned}
$$


where $\bullet^{T}$ denotes the transpose and $n_{e}$ the number of element in the patch. The components of the external force vector are

$$
\begin{aligned}
\mathrm{F}_{r}^{(G)}= & \sum_{e=1}^{n_{e}}\left[\int_{\Omega_{e}} v_{, r}\left(p^{\beta} \boldsymbol{a}_{\beta}+p^{3} \boldsymbol{a}_{3}\right) d \Omega\right. \\
& \left.+\oint_{\Gamma_{e}}\left(v_{, r}\left(p_{\tau}^{\Gamma} \boldsymbol{\tau}+p_{\nu}^{\Gamma} \boldsymbol{\nu}+p_{3}^{\Gamma} \boldsymbol{a}_{3}-\nu^{\beta}\left(\boldsymbol{a}_{3, \beta}+b_{\beta}^{\lambda} \boldsymbol{a}_{\lambda}\right) c_{\tau}^{\Gamma}\right)-v_{\beta, r} \nu^{\beta} \boldsymbol{a}_{3} c_{\tau}^{\Gamma}\right) d \Gamma\right] .
\end{aligned}
$$

Since there are 3 displacement degrees of freedom (dofs) per control point, the size of $\mathrm{K}_{r, s}^{\left(G_{4}\right)}$ and $\mathrm{F}_{r}^{(G)}$ is $3 \times 3$ and $3 \times 1$, respectively. Moreover,

$$
\begin{aligned}
& \mathcal{N}_{s}^{\beta}=\mathcal{N}^{\beta}\left(\boldsymbol{\epsilon}_{(\alpha \beta), s}, \boldsymbol{\epsilon}_{(\alpha \beta), \gamma, s}, \boldsymbol{\kappa}_{(\alpha \beta), s}, \boldsymbol{\kappa}_{(\alpha \beta), \gamma, s}\right), \\
& \mathcal{M}_{s}=\mathcal{M}\left(\boldsymbol{\epsilon}_{(\alpha \beta), s}, \boldsymbol{\epsilon}_{(\alpha \beta), \gamma, s}, \boldsymbol{\kappa}_{(\alpha \beta), s}, \boldsymbol{\kappa}_{(\alpha \beta), \gamma, s}, \boldsymbol{\kappa}_{(\alpha \beta), \gamma, \zeta, s}\right), \\
& \widetilde{\boldsymbol{n}}_{\tau, s}=\widetilde{n}_{\tau}\left(\boldsymbol{\epsilon}_{(\alpha \beta), s}, \boldsymbol{\kappa}_{(\alpha \beta), s}\right), \\
& \widetilde{\boldsymbol{n}}_{\nu, s}=\widetilde{n}_{\nu}\left(\boldsymbol{\epsilon}_{(\alpha \beta), s}, \boldsymbol{\kappa}_{(\alpha \beta), s}\right), \\
& \widetilde{\boldsymbol{n}}_{3, s}=\widetilde{n}_{3}\left(\boldsymbol{\kappa}_{(\alpha \beta), s}, \boldsymbol{\kappa}_{(\alpha \beta), \gamma, s}\right), \\
& \boldsymbol{m}_{\tau, s}=m_{\tau}\left(\boldsymbol{\kappa}_{(\alpha \beta), s}, \boldsymbol{\kappa}_{(\alpha \beta), \gamma, s}\right), \\
& \boldsymbol{m}_{\nu, s}=m_{\nu}\left(\boldsymbol{\kappa}_{(\alpha \beta), s}, \boldsymbol{\kappa}_{(\alpha \beta), \gamma, s}\right),
\end{aligned}
$$

and

$$
\begin{aligned}
\boldsymbol{\epsilon}_{(\alpha \beta), s} & =\frac{1}{2}\left(\boldsymbol{a}_{\beta} a_{\alpha, s}+\boldsymbol{a}_{\alpha} a_{\beta, s}\right), \\
\boldsymbol{\kappa}_{(\alpha \beta), s} & =-\left(\boldsymbol{a}_{3} a_{\alpha, \beta, s}+\boldsymbol{a}_{\alpha, \beta} a_{3, s}\right), \\
& =-\left(\boldsymbol{a}_{3} a_{\alpha, \beta, s}-\boldsymbol{i}_{3} \times \boldsymbol{a}_{2} a_{1, s}+\boldsymbol{i}_{3} \times \boldsymbol{a}_{1} a_{2, s}\right), \\
\boldsymbol{a}_{\alpha} & =\sum_{r=1}^{n \cdot m} R_{r, \alpha}^{p, q} \mathbf{B}_{r}, \\
a_{\alpha, s} & =R_{s, \alpha}^{p, q},
\end{aligned}
$$

where $\boldsymbol{i}_{3}=\left(\mathrm{I}-\boldsymbol{a}_{3} \boldsymbol{a}_{3}^{T}\right) \boldsymbol{a}_{\alpha, \beta} / \bar{a}_{3}$, and I is the identity matrix.

Application of the same linearization procedure to the second-order weak form (35) gives a force term identical to Eq. (42), whereas the stiffness matrix reads

$$
\mathrm{K}_{r, s}^{\left(G_{2}\right)}=\sum_{e=1}^{n_{e}} \int_{\Omega_{e}}\left(\boldsymbol{n}^{(\alpha \beta)}{ }_{, r} \boldsymbol{\epsilon}_{(\alpha \beta), s}{ }^{T}+\boldsymbol{m}^{(\alpha \beta)}{ }_{, r} \boldsymbol{\kappa}_{(\alpha \beta), s}{ }^{T}\right) d \Omega,
$$


with

$$
\begin{aligned}
& \boldsymbol{n}^{(\alpha \beta)}{ }_{, r}=n^{(\alpha \beta)}{ }_{, r}\left(\boldsymbol{\epsilon}_{(\alpha \beta), r}, \boldsymbol{\kappa}_{(\alpha \beta), r}\right), \\
& \boldsymbol{m}^{(\alpha \beta)}{ }_{, r}=m_{, r}^{(\alpha \beta)}\left(\boldsymbol{\epsilon}_{(\alpha \beta), r}, \boldsymbol{\kappa}_{(\alpha \beta), r}\right) .
\end{aligned}
$$

Considering the Galerkin approach with the Gauss-Legendre quadrature scheme, the computation of $\mathrm{K}_{r, s}^{\left(G_{2}\right)}$ is much faster than $\mathrm{K}_{r, s}^{\left(G_{4}\right)}$. Indeed, the expression of $\mathrm{K}_{r, s}^{\left(G_{2}\right)}$ is symmetric, simpler, and it requires only the computation of second-order shape-function derivatives, whereas $\mathrm{K}_{r, s}^{\left(G_{4}\right)}$ needs fourth-order ones. Moreover, $\mathrm{K}_{r, s}^{\left(G_{4}\right)}$ requires the two first derivatives of the constitutive law $\left(\mathrm{D}_{, \gamma}\right.$ and $\mathrm{D}_{, \gamma, \zeta}$, see Eq. (20)), as well as the derivatives of $a_{\alpha \beta}, a_{3}, b_{\alpha \beta}, b_{\beta}^{\alpha}$, and $\Gamma_{\alpha \beta}^{\rho}$. As shown in the next section, $\mathrm{K}_{r, s}^{\left(G_{4}\right)}$ can be collocated, which avoids the costly numerical integration steps. The question is then whether this cost reduction can compensate for the extra operations involved in the strong form.

\subsection{Collocation method}

The idea of the collocation method is to use as test functions Dirac functions $\delta$ such that

$$
\boldsymbol{v}(\xi, \eta)=\sum_{r=1}^{n \cdot m} \delta\left(\xi-\hat{\xi}_{i}\right) \delta\left(\eta-\hat{\eta}_{j}\right) \mathbf{v}_{r} ; \quad\{i ; j\}=\{1, \ldots, n ; 1, \ldots, m\},
$$

where $r=i+n(j-1)$, and $\left\{\hat{\xi}_{i}, \hat{\eta}_{j}\right\}$ are collocation point coordinates defined in Section 3.3.1. As shown in [23, 24], collocating Neumann conditions might generate some spurious oscillations in the variable field, and to overcome this issue, it is proposed to weakly enforce Neumann conditions, resulting in a hybrid collocation-Galerkin method. However, since such phenomenon has not been observed in our numerical results (which could be a consequence that only uniform parametrizations are considered), this method is not introduced here.

\subsubsection{Choice of the collocation points}

For the sake of clarity, the parametric coordinates are reported here for a univariate NURBS basis, the coordinates for a bivariate basis being obtained by considering the tensor product of two univariate ones.

Until recently, Greville abscissae (C-GP) were the most common choice of collocation point positions [21]. They are defined by the mean location of $p-1$ consecutive knots in the knot vector:

$$
\hat{\xi}_{i}=\frac{1}{p} \sum_{k=1}^{p} \xi_{i+k} ; \quad i=1, \ldots, n .
$$


Table 1: Location of the superconvergent points on the element interval $[-1,1]$ for a univariate NURBS of degree $p$ and its first to fourth derivatives.

\begin{tabular}{|c|c|c|c|c|c|}
\hline$p$ & $R^{p}$ & $R_{\alpha}^{p}$ & $R_{\alpha, \beta}^{p}$ & $R_{\alpha, \beta, \gamma}^{p}$ & $R_{\alpha, \beta, \gamma, \zeta}^{p}$ \\
\hline 1 & $-1,1$ & 0 & $\emptyset$ & $\emptyset$ & $\emptyset$ \\
\hline 2 & $-1,0,1$ & $-1,1$ & 0 & $\emptyset$ & $\emptyset$ \\
\hline 3 & $\pm \sqrt{225-30 \sqrt{30}} / 15$ & $-1,0,1$ & $\pm 1 / \sqrt{3}$ & 0 & $\emptyset$ \\
\hline 4 & $-1,0,1$ & $\pm \sqrt{225-30 \sqrt{30}} / 15$ & $-1,0,1$ & $\pm 1 / \sqrt{3}$ & 0 \\
\hline 5 & \pm 0.5049185675126533 & $-1,0,1$ & $\pm \sqrt{225-30 \sqrt{30}} / 15$ & $-1,0,1$ & $\pm 1 / \sqrt{3}$ \\
\hline 6 & $-1,0,1$ & \pm 0.5049185675126533 & $-1,0,1$ & $\pm \sqrt{225-30 \sqrt{30}} / 15$ & $-1,0,1$ \\
\hline 7 & \pm 0.503221894597504 & $-1,0,1$ & \pm 0.5049185675126533 & $-1,0,1$ & $\pm \sqrt{225-30 \sqrt{30}} / 15$ \\
\hline
\end{tabular}

However, when C-GP are used, the $L^{2}$-error decays as $\mathcal{O}\left(h^{p}\right)$ only when the basis degree is even, whereas it has recently been shown that if the degree is odd, Cauchy-Galerkin points, also called superconvergent points, should be used instead to get an optimum convergence [40, 41]. They are reported in Table 1. Note that the dimension of the spline space is lower than the total number of superconvergent points, and therefore the least-square method [40] or a subset that hits every function should be used $[41,42]$. While the subset is formed by every other point (C-ASP) in [41], resulting to a convergence of $\mathcal{O}\left(h^{p}\right)$, a better convergence order of $\mathcal{O}\left(h^{p+1}\right)$ can be obtained using clustered superconvergent points (C-CSP) [42]. Unfortunately, C-CSP are only given for second-order problems in [42]. Therefore, the present manuscript uses C-ASP points given for fourth-order problems in [41].

\subsubsection{Equation priority at the contour}

The goal of this part is to identify which equations are attributed where. Indeed, for a flat plate with only transverse displacement of the mid-surface, collocating around the contour generates $4(n+m)$ equations, whereas only $4(n+m-4)$ equations can be settled. In [38], it is proposed for clamped and simply-supported plates to reduce the number of boundary equations by averaging some of them close to the corners. We provide the generalization of this work to arbitrary boundary conditions based on the boundary condition type (Dirichlet or Neumann) and its order (the prescribed displacement, rotation, moment and shear force are composed by derivatives which are of maximum order $0,1,2$ and 3 , respectively).

In order to clarify the following explanations, all possible combinations of adjacent edges are represented in three different problems in Fig. 2. For a given plate, the $n \cdot m$ equations are represented graphically by an array of size $n \times m$. An analogy can be made between this array and the location of the control points, but a difference exists for the second row inward from the contour; the collocation 


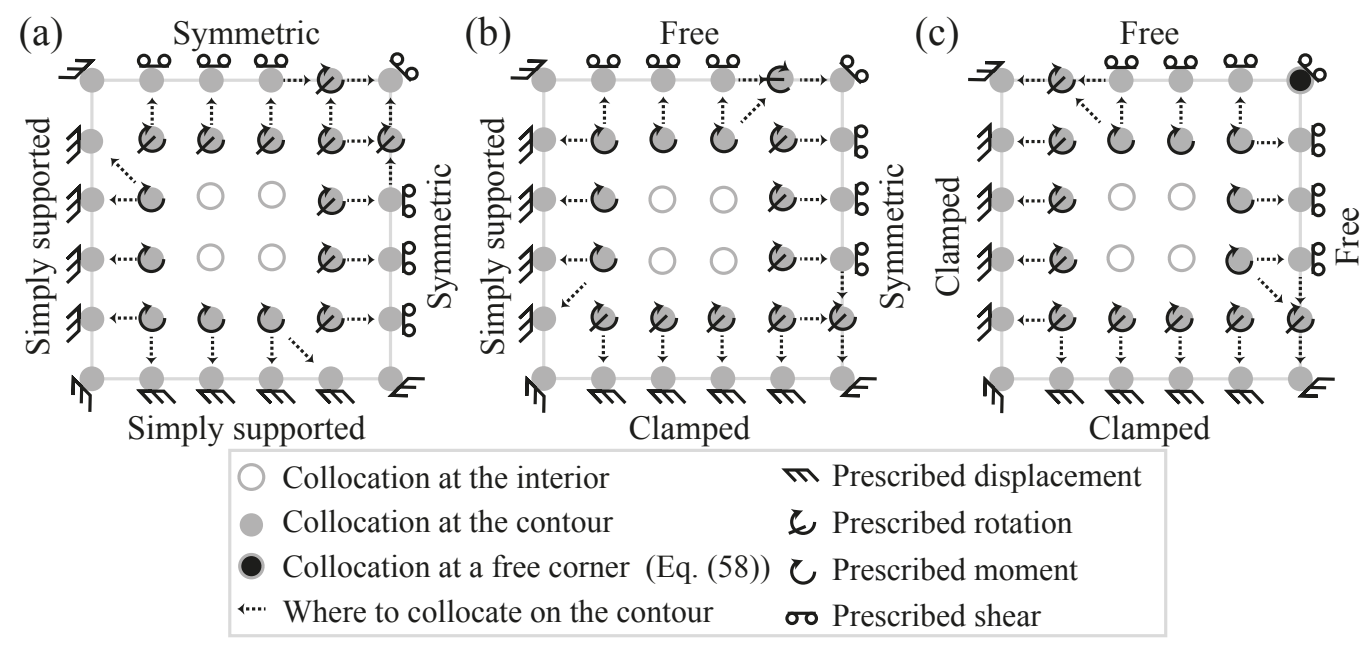

Figure 2: Three different plate problems regrouping all possible combinations of adjacent edges.

points at these locations are not considered and instead some equations evaluated at the boundary (location indicated by an arrow) are attributed to this row.

Prescribed displacement or force equations are attributed to the first row, whereas prescribed rotation or moment equations are attributed to the second row. Next to corners, two equations are attributed by default to the same location, creating a conflict. To this end, a simple rule is followed and illustrated in Fig. 3; if the two equations have the same order (e.g. they both impose a rotation), they are averaged, otherwise, one equation has the priority and the remaining one, is either averaged with an adjacent equation of the same order or discarded if evaluated at a corner. When two equations are not of the same order, if one is setting Dirichlet and the other one Neumann boundary condition, the priority is given to the Dirichlet one, whereas if they are both of Dirichlet or Neumann types, the choice of the priority has no importance. In the present work, prescribed displacements and shear forces have the priority with respect to prescribed rotations and moments, respectively, but numerical tests not reported here have shown that setting the opposite gives identical results. The performances of the proposed method is validated numerically in Section 4.2 by testing the three examples presented in Fig. 2, but before, the extension to shells is provided.

For curved shells, membrane and bending deformations are coupled such that at the second equation row around the contour, two equations originate from the interior and one from the boundary (see Fig. 4). The two equations from the interior are the components tangential to the surface, whereas the equation from 


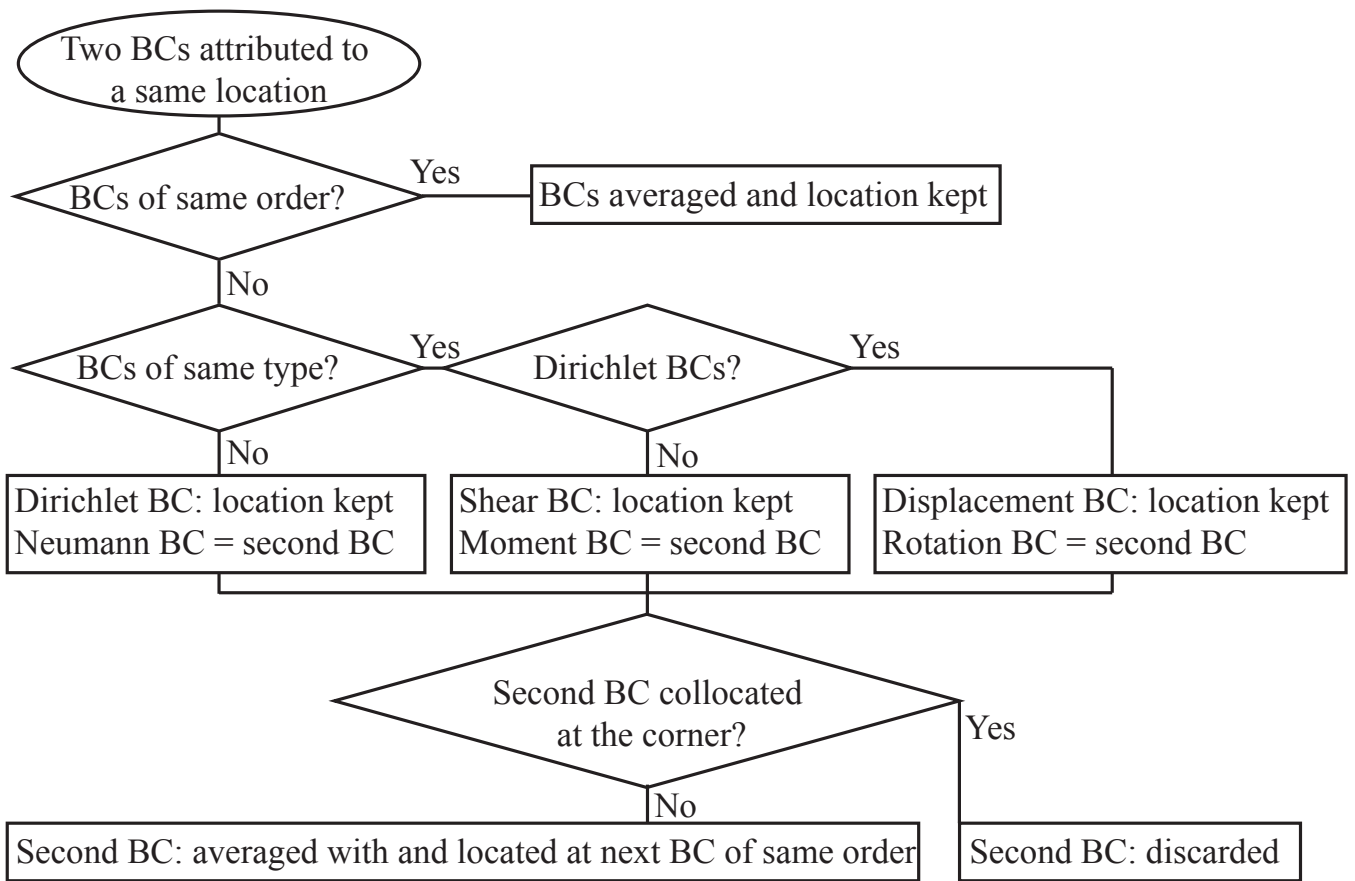

Figure 3: Collocation strategy when two boundary condition (BC) equations are attributed to a same location.

the boundary is projected to the surface normal, as indicated in the following paragraphs.

\subsubsection{Construction of the stiffness matrix and force vector}

Focusing first on the equations inside the domain, the stiffness matrix and the force vector are derived from Eq. (34) and they read

$$
\left\{\begin{array}{lr}
\mathrm{K}_{r, s}^{(\mathrm{Int})}=-\left.\left(\boldsymbol{a}_{\beta} \mathcal{N}_{s}^{\beta^{T}}+\boldsymbol{a}_{3} \mathcal{M}_{s}^{T}\right)\right|_{\left\{\hat{\xi}_{i}, \hat{\eta}_{j}\right\}} & \{i ; j\}=\{3, \ldots, n-2 ; 3, \ldots, m-2\}, \\
\mathrm{F}_{r}^{(\mathrm{Int})}=\left.\left(\boldsymbol{a}_{\beta} p^{\beta}+\boldsymbol{a}_{3} p^{3}\right)\right|_{\left\{\hat{\xi}_{i}, \hat{\eta}_{j}\right\}} & \{i ; j\}=\{2, \ldots, n-1 ;\{2, m-1\}\} \\
\mathrm{K}_{r, s}^{(\mathrm{Int})}=-\left.\left(\boldsymbol{a}_{\beta} \mathcal{N}_{s}^{\beta^{T}}\right)\right|_{\left\{\hat{\xi}_{i}, \hat{\eta}_{j}\right\}} & \cup\{\{2, n-1\} ; 2, \ldots, m-1\} . \\
\mathrm{F}_{r}^{(\mathrm{Int})}=\left.\left(\boldsymbol{a}_{\beta} p^{\beta}\right)\right|_{\left\{\hat{\xi}_{i}, \hat{\eta}_{j}\right\}} &
\end{array}\right.
$$




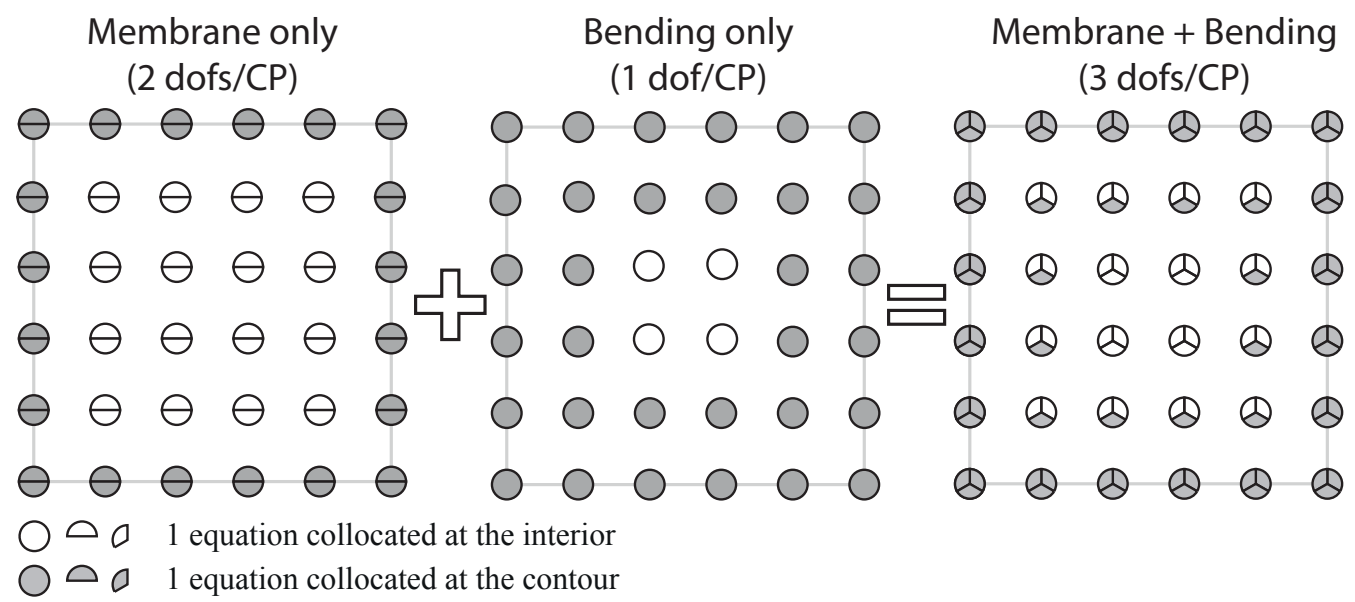

Figure 4: For each collocation point (CP), the number of empty and full circle portions indicates the number of equations collocated inside and at the contour of the domain, respectively.

Concerning the collocation of the boundary conditions, if the displacement tangential to the edge surface is provided,

$$
\left\{\begin{array}{lrl}
\mathrm{K}_{r, s}^{(\mathrm{BC} \tau)}=\left.\left(\boldsymbol{\tau} \boldsymbol{u}_{\tau, s}^{T}\right)\right|_{\left\{\hat{\xi}_{i}, \hat{\eta}_{j}\right\}} & \{i ; j\}=\{1, \ldots, n ;\{1, m\}\} \\
\mathrm{F}_{r}^{(\mathrm{BC} \tau)}=\left.\left(\boldsymbol{\tau} u_{\tau}^{\Gamma}\right)\right|_{\left\{\hat{\xi}_{i}, \hat{\eta}_{j}\right\}} & \cup\{\{1, n\} ; 1, \ldots, m\},
\end{array}\right.
$$

whereas if the tangential displacement is free,

$$
\left\{\begin{array}{lrl}
\mathrm{K}_{r, s}^{(\mathrm{BC} \tau)}=\left.\left(\boldsymbol{\tau} \boldsymbol{n}_{\tau, s}^{T}\right)\right|_{\left\{\hat{\xi}_{i}, \hat{\eta}_{j}\right\}} & \{i ; j\}=\{1, \ldots, n ;\{1, m\}\} \\
\mathrm{F}_{r}^{(\mathrm{BC} \tau)}=\left.\left(\boldsymbol{\tau} p_{\tau}^{\Gamma}\right)\right|_{\left\{\hat{\xi}_{i}, \hat{\eta}_{j}\right\}} & \cup\{\{1, n\} ; 1, \ldots, m\} .
\end{array}\right.
$$

Similarly, a Dirichlet boundary condition to the direction tangential to the surface but normal to the edge reads

$$
\left\{\begin{array}{lrl}
\mathrm{K}_{r, s}^{(\mathrm{BC} \nu)}=\left.\left(\boldsymbol{\nu} \boldsymbol{u}_{\nu, s}^{T}\right)\right|_{\left\{\hat{\xi}_{i}, \hat{\eta}_{j}\right\}} & \{i ; j\} & =\{1, \ldots, n ;\{1, m\}\} \\
\mathrm{F}_{r}^{(\mathrm{BC} \nu}=\left.\left(\boldsymbol{\nu} u_{\nu}^{\Gamma}\right)\right|_{\left\{\hat{\xi}_{i}, \hat{\eta}_{j}\right\}} & \cup\{\{1, n\} ; 1, \ldots, m\},
\end{array}\right.
$$

whereas imposing a Neumann boundary condition gives:

$$
\left\{\begin{array}{lrl}
\mathrm{K}_{r, s}^{(\mathrm{BC} \nu)}=\left.\left(\boldsymbol{\nu} \boldsymbol{n}_{\nu, s}^{T}\right)\right|_{\left\{\hat{\xi}_{i}, \hat{\eta}_{j}\right\}} & \{i ; j\} & =\{1, \ldots, n ;\{1, m\}\} \\
\mathrm{F}_{r}^{(\mathrm{BC} \nu)}=\left.\left(\boldsymbol{\nu} p_{\nu}^{\Gamma}\right)\right|_{\left\{\hat{\xi}_{i}, \hat{\eta}_{j}\right\}} & \cup\{\{1, n\} ; 1, \ldots, m\} .
\end{array} .\right.
$$


For the case of a given transverse displacement, one reads

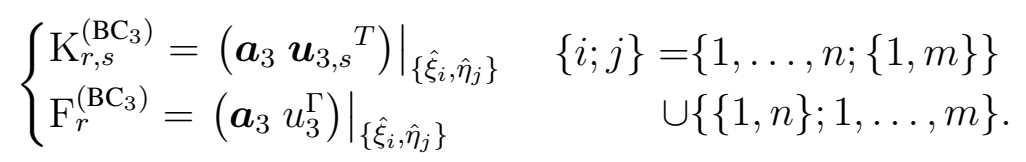

For a prescribed rotation

$$
\left\{\begin{array}{lrl}
\mathrm{K}_{r, s}^{\left(\mathrm{BC}_{3}\right)}=\left.\left(\boldsymbol{a}_{3} \boldsymbol{\omega}_{\tau, s}^{T}\right)\right|_{\left\{\hat{\xi}_{k}, \hat{\eta}_{l}\right\}} & \{i ; j ; k ; l\}=\left\{1, \ldots, n ;\{2, m-1\} ; i ; l_{j}\right\} \\
\mathrm{F}_{r}^{\left(\mathrm{BC}_{3}\right)}=\left.\left(\boldsymbol{a}_{3} \omega_{\tau}^{\Gamma}\right)\right|_{\left\{\hat{\xi}_{k}, \hat{\eta}_{l}\right\}} & \cup\left\{\{2, n-1\} ; 1, \ldots, m ; k_{i} ; j\right\},
\end{array}\right.
$$

and for a prescribed moment,

$$
\left\{\begin{array}{lr}
\mathrm{K}_{r, s}^{\left(\mathrm{BC}_{3}\right)}=\left.\left(\boldsymbol{a}_{3} \boldsymbol{m}_{\tau, s}\right)\right|_{\left\{\hat{\xi}_{k}, \hat{\eta}_{l}\right\}} & \{i ; j ; k ; l\}=\left\{2, \ldots, n-1 ;\{2, m-1\} ; i ; l_{j}\right\} \\
\mathrm{F}_{r}^{\left(\mathrm{BC}_{3}\right)}=\left.\left(\boldsymbol{a}_{3} c_{\tau}^{\Gamma}\right)\right|_{\left\{\hat{\xi}_{k}, \hat{\eta}_{l}\right\}} & \cup\left\{\{2, n-1\} ; 2, \ldots, m-1 ; k_{i} ; j\right\},
\end{array}\right.
$$

where

$$
k_{i}=\left\{\begin{array}{l}
1 \text { if } i=2, \\
n \text { if } i=n-1,
\end{array} l_{j}=\left\{\begin{aligned}
1 & \text { if } j=2, \\
m & \text { if } j=m-1 .
\end{aligned}\right.\right.
$$

Finally, for a prescribed shear force not located at a free corner

$$
\left\{\begin{array}{lr}
\mathrm{K}_{r, s}^{\left(\mathrm{BC}_{3}\right)}=\left.\left(\boldsymbol{\nu} \widehat{\boldsymbol{n}}_{\nu, s}^{T}+\boldsymbol{\tau} \widehat{\boldsymbol{n}}_{\tau, s}^{T}+\boldsymbol{a}_{3} \widehat{\boldsymbol{n}}_{3, s}^{T}\right)\right|_{\left\{\hat{\xi}_{i}, \hat{\eta}_{j}\right\}} & \{i ; j\}=\{1, \ldots, n ;\{1, m\}\} \\
\mathrm{F}_{r}^{\left(\mathrm{BC}_{3}\right)}=\left.\left(\boldsymbol{a}_{3} p_{3}^{\Gamma}\right)\right|_{\left\{\hat{\xi}_{i}, \hat{\eta}_{j}\right\}} & \cup\{\{1, n\} ; 1, \ldots, m\},
\end{array}\right.
$$

whereas at free corners (intersection of two adjacent free-free edges),

$$
\left\{\begin{array}{l}
\mathrm{K}_{r, s}^{\left(\mathrm{BC}_{3}\right)}=\left.\left(\left[\boldsymbol{a}_{3} \boldsymbol{m}_{\nu}^{T}\right]_{\mathrm{C}^{-}}^{\mathrm{C}^{+}}\right)\right|_{\left\{\hat{\xi}_{i}, \hat{\eta}_{j}\right\}} \quad\{i ; j\}=\{\{1, n\} ;\{1, m\}\} . \\
\mathrm{F}_{r}^{\left(\mathrm{BC}_{3}\right)}=\left.\left(\boldsymbol{a}_{3} p_{3}^{\Gamma}\right)\right|_{\left\{\hat{\xi}_{i}, \hat{\eta}_{j}\right\}}
\end{array}\right.
$$

The total stiffness matrix and force vector are then the sum of the different contributions:

$$
\left\{\begin{array}{l}
\mathrm{K}^{(C)}=\mathrm{K}^{(\mathrm{Int})}+\mathrm{K}^{\left(\mathrm{BC}_{\tau}\right)}+\mathrm{K}^{\left(\mathrm{BC}_{\nu}\right)}+\mathrm{K}^{\left(\mathrm{BC}_{3}\right)}+\mathrm{K}^{\left(\mathrm{BC}_{3}\right)}, \\
\mathrm{F}^{(C)}=\mathrm{F}^{(\mathrm{Int})}+\mathrm{F}^{\left(\mathrm{BC}_{\tau}\right)}+\mathrm{F}^{\left(\mathrm{BC}_{\nu}\right)}+\mathrm{F}^{\left(\mathrm{BC}_{3}\right)}+\mathrm{F}^{\left(\mathrm{BC}_{3}\right)}
\end{array}\right.
$$

Note that $\mathrm{K}^{\left(\mathrm{BC}_{3}\right)}$ and $\mathrm{F}^{\left(\mathrm{BC}_{3}\right)}$ are written twice since in the transverse direction of the surface, two boundary conditions are required. 


\section{Numerical results}

Before investigating the collocation method, the implementation of the stiffness matrix including the effects of the boundary conditions has been tested on all the examples which are treated in the following section, by making sure that the Galerkin method applied to the fourth-order problem is identical to the secondorder one (i.e. Eq. (41) yields identical results as Eq. (45)). It is worth noting that in order to get an equality up to the machine precision, a large number of Gauss quadrature points should be selected, especially for coarse meshes). For instance, for the problem presented in Section 4.3 with the same mesh used to plot Fig. 9a-b, with $p+3$ Gauss points per element and per direction, it is found that $\max \left|\mathrm{K}^{\left(G_{4}\right)}-\mathrm{K}^{\left(G_{2}\right)}\right| / \max \left|\mathrm{K}^{\left(G_{2}\right)}\right|<1.10^{-13}$.

In what follows, for the sake of simplicity, the degrees of the NURBS are considered identical in the two parametric directions $(p=q)$. We denote by IGA$\mathrm{G}$ the classical Galerkin method applied to the second-order weak form (Eq. (45)), whereas IGA-C refers to the collocation method using C-GP and C-ASP for even and odd $p$, respectively. "C", "S", "G" and "F" denote the clamped, simplysupported, symmetric (guided) and free boundary conditions, respectively. All simulations in this work are performed with an in-house MATLAB code that makes use of some functionalities of the open source NURBS toolbox [46, 47].

This section applies the collocation method to different benchmarks, involving in a first part rectangular, circular and annular flat plates, and in a second part curved shells. But before presenting the results, an a-priori $L^{2}$-error estimate is introduced, which will be used to validate the convergence orders.

\subsection{A-priori error estimation: convergence order}

Convergence plots under $h$-refinement are obtained by computing the approximated solution and the exact one of a PDE problem, and the convergence order can be verified by using a-priori error estimates for instance in the $L^{2}\left(L^{2} \equiv H^{0}\right)$ error norm [6]:

$$
\left\|\boldsymbol{u}-\boldsymbol{u}_{h}\right\|_{L^{2}(\Omega)}=\left(\int\left(\boldsymbol{u}-\boldsymbol{u}_{h}\right)^{2} d \Omega\right)^{1 / 2} .
$$

When considering PDEs of order $2 m$ with $m \geq 1$ and its exact solution $\boldsymbol{u} \in$ $H^{r}(\Omega)$ ( $r$ is the regularity), the error in the norm $L^{2}$ for a $\mathcal{C}^{p-1}$-continuous NURBS basis can be estimated as:

$$
\left\|\boldsymbol{u}-\boldsymbol{u}_{h}\right\|_{L^{2}(\Omega)}^{2} \leq C h^{\beta}\|\boldsymbol{u}\|_{H^{r}(\Omega)},
$$


Table 2: Estimated convergence orders $\beta$ in the $L^{2}$ norm, for a globally $\mathcal{C}^{p-1}$-continuous NURBS basis of degree $p$, and for a PDE problem of order $2 m$ with $r=\infty$.

\begin{tabular}{|c|c||c|c|c|c|c|c|c|}
\hline & $m \backslash p$ & 1 & 2 & 3 & 4 & 5 & 6 & 7 \\
\hline \hline$\beta_{\text {IGA-G }}$ & 1 & 2 & 3 & 4 & 5 & 6 & 7 & 8 \\
\hline$\beta_{\text {IGA-G }}$ & 2 & 0 & 2 & 4 & 5 & 6 & 7 & 8 \\
\hline \hline$\beta_{\text {IGA-C }}$ & 1 & 0 & 2 & 3 & 4 & 5 & 6 & 7 \\
\hline$\beta_{\text {IGA-C }}$ & 2 & 0 & 0 & 0 & 2 & 3 & 4 & 5 \\
\hline
\end{tabular}

where $h$ is the characteristic mesh size of the elements, and $C$ a constant independent of $\boldsymbol{u}$ and $h ; \beta$ gives a lower bound for the convergence order estimate and depends on the discretization procedure. When the PDE is solved using the Galerkin method [6],

$$
\beta_{I G A-G}=\min \{\delta, 2(\delta-m)\},
$$

with $\delta=\min \{r, p+1\}$. For the collocation method, a-priori error estimates are really challenging and where only proved so far for one-dimensional second-order problems with C-GP [21]. When the collocation method is used with C-GP and CASP for even and odd $p$, respectively, we state based on observations and without proving it in a mathematical framework that

$$
\beta_{I G A-C}=\delta-(2 m-1) .
$$

While for $m=1$, this statement can be found in [21, 22, 41, 42, 48], for $m=2$ this estimate is only based on the convergence order observed and reported in [38, 41], and confirmed by the present work. Estimated convergence orders for NURBS basis of degree up to $p=7$ are given in Table 2 for both discretization methods, for second as well as fourth-order problems. Note that the values reported in this table assume that the regularity of the exact deflection is infinite, which is not valid for some Kirchhoff-Love shell configurations.

Indeed, it is demonstrated in [49-51] that at a corner of a Kirchhoff-Love plate, when its surrounding is loaded by a pressure (not a point force), the exact deflection has a critical regularity $r\left(u_{3} \in H^{r}\right)$. This value is reported in Table 3 for different adjacent boundary conditions. In addition, the corresponding estimated convergence orders are reported for $r<p+1$, and they are going to be used next to validate a set of different benchmarks.

\subsection{Flat plates}

These first examples investigate flat plates to validate the collocation strategy. As this decouples the membrane and bending deformations, only the transverse 
Table 3: The critical corner regularity $r\left(u_{3} \in H^{r}\right)$ for $\nu=0.3$, an angle of $90^{\circ}$, and different adjacent edge boundary conditions [49]. Estimated convergence orders $\beta$ in the $L^{2}$ norm, assuming $r<p+1$ and $m=2$.

\begin{tabular}{|c||c|c|c|c|c|}
\hline Adjacent edges & CC & CS & CF & SS, SF & FF \\
\hline$r$ & 4.74 & 4 & 3.07 & 3 & 3.76 \\
\hline$\beta_{I G A-G}$ & $\min \{4.74,5.48\}$ & 4 & $\min \{2.14,3.07\}$ & $\min \{2,3\}$ & $\min \{3.52,3.76\}$ \\
\hline$\beta_{I G A-C}$ & 1.74 & 1 & 0.07 & 0 & 0.76 \\
\hline
\end{tabular}

displacements are considered here, as thus component dominates structural response of the structural member. The bending stiffness is $D=E t^{3} / 12 /\left(1-\nu^{2}\right)=$ $1 \mathrm{Nm}$ and the Poisson ratio $\nu=0.3$. Moreover, for all benchmarks involving a uniform loading, the pressure is $p_{3}=1 \mathrm{Nm}^{-2}$.

\subsubsection{Square plates under distributed loads}

A fully-clamped and a fully simply-supported square plate $\left([0 L]^{2}, L=1 \mathrm{~m}\right)$ are first considered under a sinusoidal loading, such that for the clamped plate, the pressure and the resulting deflection are given respectively by [44]

$$
\begin{aligned}
& p_{3}(x, y)=\left(1-\cos \frac{2 \pi x}{L}\right)\left(1-\cos \frac{2 \pi y}{L}\right), \\
& u_{3}(x, y)=-\frac{16 \pi^{4}}{D L^{4}}\left(\cos \frac{2 \pi x}{L}-4 \cos \frac{2 \pi x}{L} \cos \frac{2 \pi y}{L}+\cos \frac{2 \pi y}{L}\right),
\end{aligned}
$$

whereas for the fully simply-supported plate

$$
\begin{aligned}
& p_{3}(x, y)=\sin \frac{2 \pi x}{L} \sin \frac{2 \pi y}{L}, \\
& u_{3}(x, y)=-\frac{16 \pi^{4}}{D L^{4}} \sin \frac{2 \pi x}{L} \sin \frac{2 \pi y}{L} .
\end{aligned}
$$

Taking advantage of symmetry, only one quarter of the plate is modeled, resulting in the plate problems CCGG and SSGG. Convergence curves under $h$-refinement for different NURBS degree $p$ are shown in Figs. 5a-b. For IGA-G and IGA-C, it is observed that the convergence orders are in good agreement with Eqs. (62) and (63), respectively (see also Table 2), such that for IGA-C, this confirms the previous convergence orders reported in the literature [38, 41] and from which Eq. (63) has been stated.

We consider now exactly the same problems, except that the sinusoidal loading is replaced by a uniform pressure. Analytical solutions for both problems 
can be found in [52] and they are expressed as a sum of a particular $\left(u_{\mathrm{par}}\right)$ and a homogeneous $\left(u_{\text {hom }}\right)$ solution such that

$$
u_{3}(x, y)=u_{\mathrm{par}}(x)+u_{\mathrm{hom}}(x, y)
$$

where

$$
\begin{aligned}
u_{\text {hom }}(x, y)=\frac{p_{3}}{D} \sum_{n=0}^{\infty}(-1)^{n} & \left(\left(A_{n} \frac{\cosh \alpha_{n} y^{\prime}}{\cosh \alpha_{n} a}+B_{n} \frac{y^{\prime}}{a} \frac{\sinh \alpha_{n} y^{\prime}}{\cosh \alpha_{n} a}\right) \cos \alpha_{n} x^{\prime}\right. \\
+ & \left.\left(C_{n} \frac{\cosh \alpha_{n} x^{\prime}}{\cosh \alpha_{n} a}+D_{n} \frac{x^{\prime}}{a} \frac{\sinh \alpha_{n} x^{\prime}}{\cosh \alpha_{n} a}\right) \cos \alpha_{n} y^{\prime}\right),
\end{aligned}
$$

with $\alpha_{n}=(2 n+1) \pi / L, a=L / 2, x^{\prime}=x-L / 2$, and $y^{\prime}=y-L / 2$. For the fully simply-supported plate,

$$
u_{\mathrm{par}}(x)=\frac{p_{3}}{24 D}\left(x^{4}-6 a^{2} x^{2}+5 a^{4}\right),
$$

and

$$
A_{n}=-B_{n}\left(\tanh \alpha_{n} a+\frac{2}{\alpha_{n} a}\right), B_{n}=\frac{1}{\alpha_{n}^{4}}, C_{n}=D_{n}=0,
$$

whereas for the fully clamped plate, different coefficients hold for $A_{n}, B_{n}, C_{n}$, $D_{n}$ and $u_{\text {par }}$ [52] (not reported here for the sake of brevity). Convergence plots are shown in Figs. 5c-d, and convergence orders tend towards the ones reported in Table 2, up to $p=4$ for IGA-G and up to $p=6$ for IGA-C. For $p \geq 5$ (IGA-G) and $p \geq 7$ (IGA-C), the convergence orders do not increase with $p$, which can be explained by the critical regularity of the exact solution (Table 3 ). Moreover, the convergence orders are found to be superconvergent with respect to their estimates, in agreement with the a-priori error estimate given in Eq. (63).

A uniformly loaded CSFS plate is then considered (analytical solution in [53] $]^{1}$ ), which is reduced using symmetries to the CSFG plate presented in Fig. 2b. Similarly to previous examples with uniform loading, convergence orders (Fig. 6a) are the estimated ones with $r=\infty$ up to $p=4$ for IGA-G and $p=6$ for IGA-C. For

\footnotetext{
${ }^{1}$ In [53], concerning the CSFS plate, $\alpha$ in the nominator of Eq. (38) should be replaced by $a$, and in Eq. (54), $\sum_{n=1}^{\infty}$ should be replaced by $\sum_{n=1,3,5, \ldots}^{\infty}$.
} 

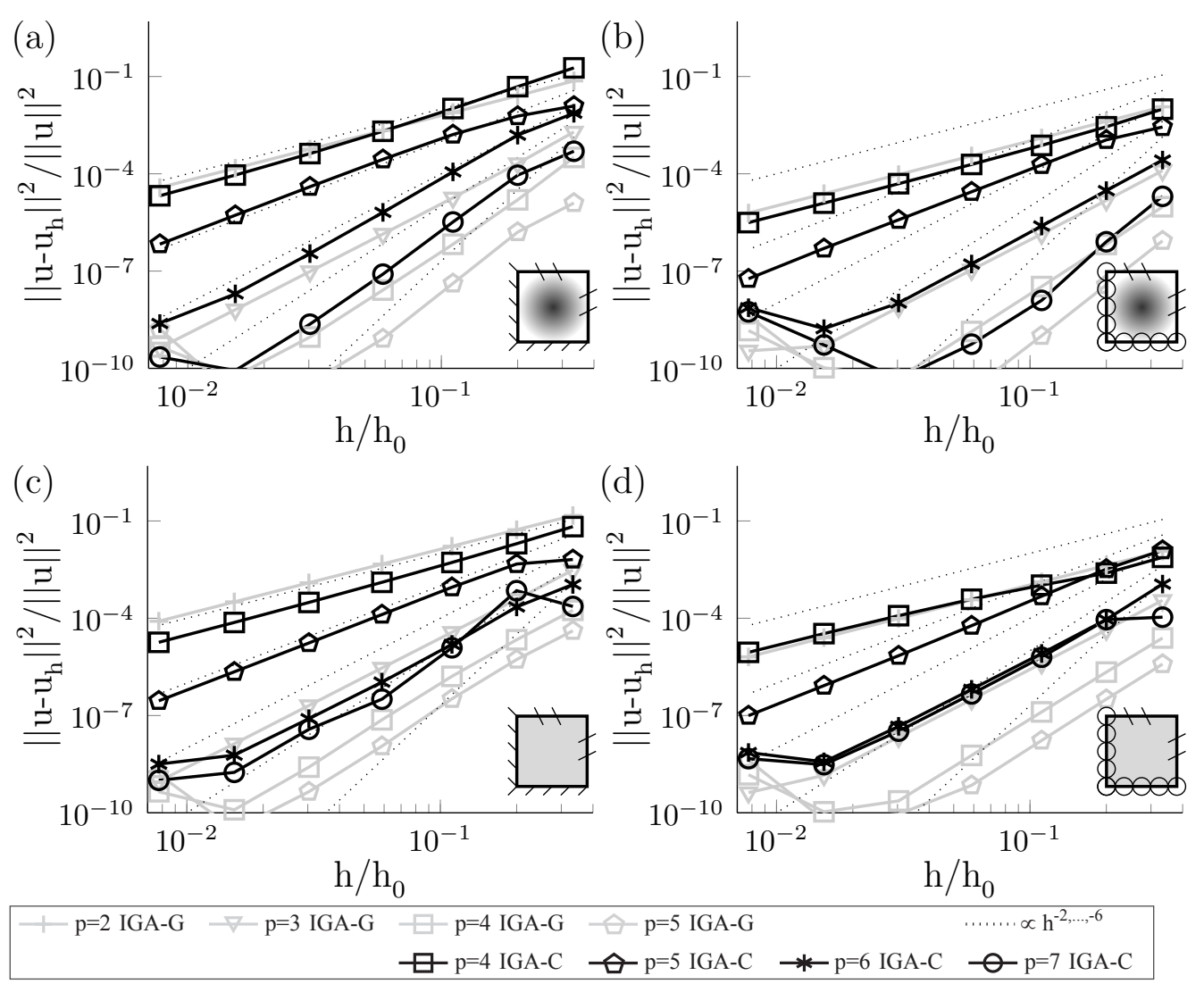

Figure 5: Relative $L^{2}$-error versus normalized mesh size $h / h_{0}$ for the CCGG $(\mathrm{a}, \mathrm{c})$ and the SSGG $(\mathrm{b}, \mathrm{d})$ square plates under sinusoidal $(\mathrm{a}, \mathrm{b})$ and uniform $(\mathrm{c}, \mathrm{d})$ loading.

higher degrees, the convergence orders remain constant and they are superconvergent with respect to the estimated ones when considering the corner regularity $(r=3$ for adjacent SF edges in Table 3).

Up to this point, all possible combinations of adjacent edges have been validated, except two of them: adjacent FF edges and adjacent CF edges. The free corner is investigated by studying a SSFF plate under a uniform pressure [54 $]^{2}$. Convergence curves are shown in Fig. 6b, and the convergence orders are 0.76 for IGA-C, and $2(p=2)$ or $3.76(p \geq 3)$ for IGA-G. These values are relatively low, but they coincide with the estimated ones reported for adjacent FF edges (Table 3 ).

The remaining boundary combination is the adjacent CF edges, which is in-

\footnotetext{
${ }^{2}$ In [54], $\pi$ should be replaced by $\pi^{3}$ in the denominator of Eqs. (48) and (49).
} 
vestigated from the uniformly loaded CCFF plate presented in Fig. 2c (analytical solution in [54]). Convergence plots are shown in Fig. 6c, and whatever the degree $p$ is, convergence orders do not converge to constant values. One possible reason is the following; focusing on the free edge, the torsional component of the moment at the contour $\left(m_{\nu}\right)$ is not zero at the clamped corner, whereas it is zero just beyond it (along the free edge). This means that $m_{\nu}$ is discontinuous, and so its first derivative, required in the Kirchhoff-Love theory (see Eq. (30)), is locally not defined. The convergence is then driven by this ill-conditioning, and not by the mesh size. Anyway, the convergence orders are superconvergent with respect to their estimates $\left(\beta_{I G A-G}=2.14\right.$ and $\left.\beta_{I G A-C}=0.07\right)$. Note that for a CFCF plate (analytical solution in [52]), only adjacent clamped-free edges are involved and the convergence plots are similar - although they are not reported here for the sake of brevity.

Finally, a uniformly loaded square plate supported at its corners is investigated, for which an analytical solution can be found in [55]. To the best of the authors' knowledge, regularity of the deflection at the corners for this configuration has not been investigated yet, and we simply note that the convergence orders (Fig. 6d) are higher than the ones reported for a plate with a free corner (SSFF, see Fig. 6b).

\subsubsection{Square plates under point loads}

Imposing point loads with the collocation method is not straight forward [28, 39] and the strategy used in this work is discussed next, but before, the particular case of a point load applied at a free-free corner is presented.

At a free corner, a singularity in the shear force field exists and is quantified by Eq. (58), such that this equation can be directly used to balance a concentrated load. Using the fact that at the free corner of a unit-length square plate, the punctual shear force is $F_{C}=-2 D(1-\nu) \frac{\partial^{2} u_{3}}{\partial x \partial y}$ [44], an analytical solution of the deflection for a square plate simply supported at the sides $x=0$ and $y=0$, and free at $x=1$ and $y=1$ with a prescribed corner force $F_{C}$ at $\{x, y\}=\{1,1\}$ can be computed easily:

$$
u_{3}(x, y)=\frac{-F_{C}}{2 D(1-\nu)} x y
$$

Since this solution is only a second-order polynomial, it is verified that the numerical solution is patch-test compliant (i.e. the machine precision is obtained for all mesh size).

In order to analyze the more general case of a point load not located at a free corner, the fully simply supported square plate with a point load $\left(F_{M}\right)$ in its middle 

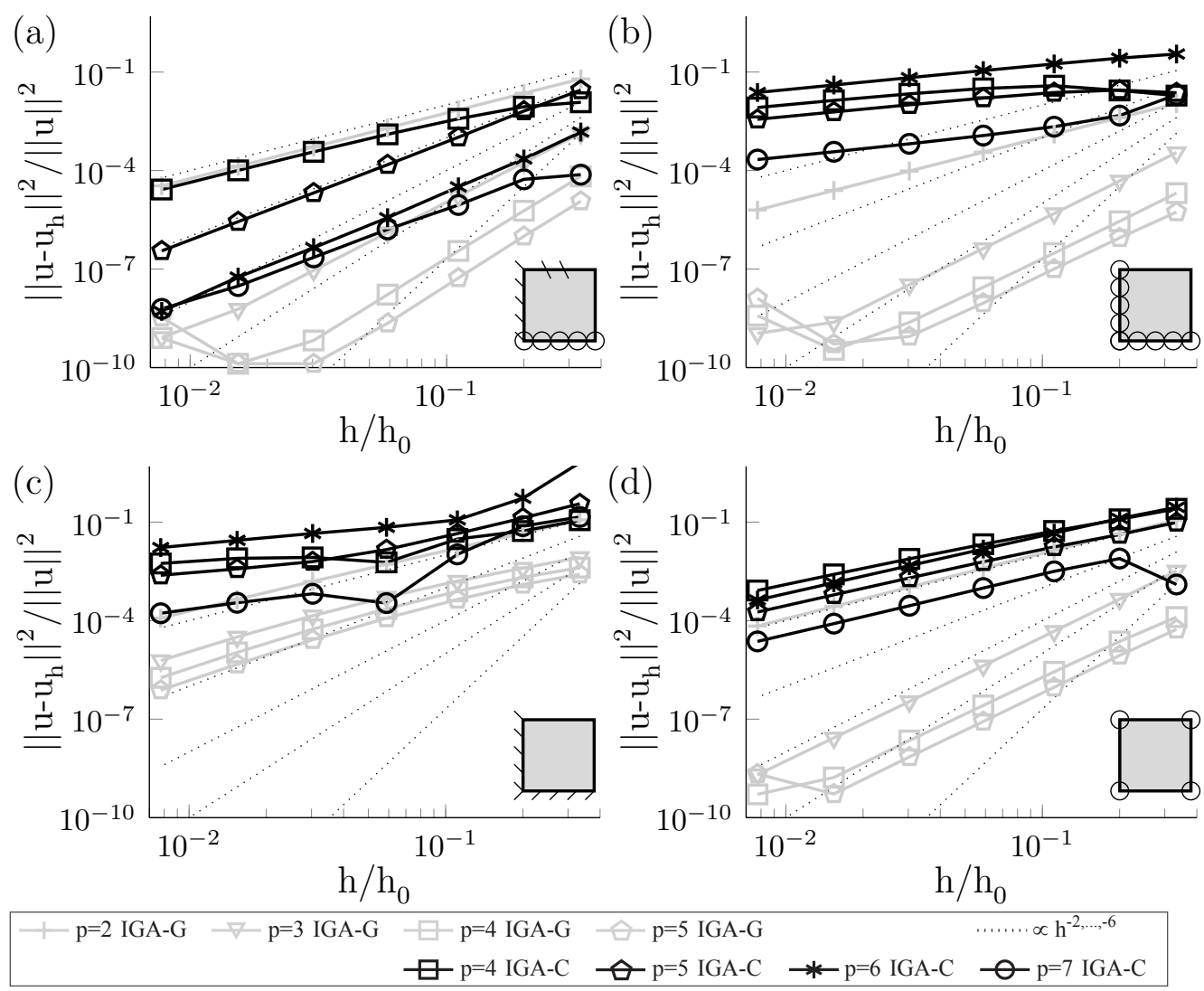

Figure 6: Relative $L^{2}$-error versus normalized mesh size $h / h_{0}$ for uniformly loaded CSFG (a), SSFF (b), CCFF (c) and corner-supported FFFF (d) square plates.

is investigated. The plate deflection is given by [56]

$$
u_{3}(x, y)=-\frac{4 F_{M} L^{2}}{D \pi^{4}} \sum_{n=1}^{\infty} \sum_{m=1}^{\infty} \frac{\sin \frac{n \pi}{2} \sin \frac{m \pi}{2} \sin \frac{n \pi x}{L} \sin \frac{m \pi y}{L}}{\left(n^{2}+m^{2}\right)^{2}} .
$$

To process the singularity, the strategy developed in [39] is used which assumes that a collocation point $\left(\hat{\xi}_{k}, \hat{\eta}_{l}\right)$ is present at the location of the point force. Assuming the pressure at the collocation points to be

$$
p_{3}^{h}\left(\hat{\xi}_{i}, \hat{\eta}_{j}\right)=\left\{\begin{array}{l}
\frac{F_{M}}{\lambda_{p}} \text { if } i=k \text { and } j=l, \\
0 \text { otherwise }
\end{array}\right.
$$

solving this system gives the discrete pressure field $p_{3}^{h}$, where the scaling constant 
$\lambda_{p}$ is determined after integrating

$$
\left.\int_{\Omega} p_{3}^{h}\right|_{\lambda_{p}=1} d \Omega=\lambda_{p}
$$

The convergence curves are shown in Fig. 7a for both the collocation and the Galerkin methods, and it is observed that the maximum convergence order is 3 , driven by the load singularity (it is 2 for IGA-G $p=2$ and for IGA-C $p=4$ in agreement with Table 2). Note that for a uniform mapping, any C-ASP is located at the mid-span, justifying why the collocation method with the order $p=5,7$ are not provided in this analysis. This limitation could have been removed by distributing the concentrated load to several collocation points located around the mid-span, as proposed in [28], but is not considered here for the sake of brevity.

Since the problem is symmetric, this case study is reconsidered restricting the analysis to one quarter of the plate. Consequently, the point force is now located at a non-free corner. At this location and along the sides, the shear force has the unit of a linear pressure, which may suggest that the same strategy applied to the pressure field can be used to obtained the linear shear force. However, there are two major differences:

- The linear shear force is not defined everywhere a priori: there are more equations than unknowns. Indeed, as shown in Fig. 2a, at a corner of adjacent symmetric edges, two times two equations describing shear forces are averaged. It means that in order to get the shear force at those positions, a "scaled" displacement field has to be computed first from which a "scaled"shear force field can be retrieved.

- The pressure is zero at the collocation points inside the plate, but is not defined at its contour. Again, it can only be computed a posteriori from the scaled displacement field.

The proposed strategy is then the following. Denoting $\left(\hat{\xi}_{n}, \hat{\eta}_{m}\right)$ the collocation point at the corner, the shear forces and the pressure field are respectively defined by

$$
\begin{gathered}
p_{3}^{\Gamma, h}\left(\hat{\xi}_{i}, \hat{\eta}_{j}\right)=\left\{\begin{array}{rr}
\frac{F_{C}}{\lambda} \text { if } i=n \text { and } j=m, \quad\{i ; j\}=\{1, \ldots, n ; m\} \\
0 \text { otherwise }, & \cup\{n ; 1, \ldots, m\},
\end{array}\right. \\
p_{3}^{h}\left(\hat{\xi}_{i}, \hat{\eta}_{j}\right)=0, \quad\{i ; j\}=\{3, \ldots, n-2 ; 3, \ldots, m-2\},
\end{gathered}
$$


where $F_{C}=F_{M} / 4$. Since $\lambda$ is not known a priori, an auxiliary problem $\left(\left.u_{3}^{h}\right|_{\lambda=1}\right)$ is solved choosing $\lambda=1$. Using Eqs. (31) and (33), the resulting total pressure and shear force are respectively

$$
\begin{aligned}
& \int_{\Gamma} \boldsymbol{a}_{3} \widehat{\boldsymbol{n}}_{3}\left(\left.u_{3}^{h}\right|_{\lambda=1}\right) d \Gamma=\lambda_{s}, \\
& \int_{\Omega} \boldsymbol{a}_{3} \boldsymbol{\mathcal { M }}^{T}\left(\left.u_{3}^{h}\right|_{\lambda=1}\right) d \Omega=\lambda_{p} .
\end{aligned}
$$

Finally, one gets $\lambda=\lambda_{s}+\lambda_{p}$, and since the problem has been assumed linear in the present manuscript (small deformations), $u_{3}^{h}=\left.u_{3}^{h}\right|_{\lambda=1} / \lambda$.

The convergence curves are shown in Fig. $7 \mathrm{~b}$ and all the convergence orders are found to be equal to 2 for IGA-C and IGA-G with $p=2$, whereas it is 3 for IGA-G with $p>2$. Moreover, for IGA-C, it is observed that the error is smaller for basis of even degrees than for odd ones: C-ASP might not be optimum for the enforcement of point loads.

While results converge, a critical drawback of the proposed method is the computation time, as it is going to be shown in Section 4.5. Indeed, the term $\mathcal{M}$ involves fourth-order derivatives of the displacement field, and needs to be integrated. A first solution to save some computation time would consist in using a reduced Gauss-Lobatto quadrature rules for the numerical integration, as proposed in [57]. Another idea is to limit the integration area around the point of the load application and could be the topic of future research.

\subsubsection{Circular plates}

In this section, fully clamped and simply supported versions of a uniformly load circular plate of radius $R_{o}=1 \mathrm{~m}$ are investigated. Analytical solutions can be found in [44], and the deflection at a distance $R$ from the center for the clamped case reads

$$
u_{3}(R)=\frac{p_{3}}{64 D}\left(R_{o}^{2}-R^{2}\right)^{2}
$$

whereas for the simply-supported circular plate

$$
u_{3}(R)=\frac{p_{3} R_{o}^{4}}{64 D}\left(\frac{R^{4}}{R_{o}^{4}}-2 \frac{3+\nu}{1+\nu} \frac{R^{2}}{R_{o}^{2}}+\frac{5+\nu}{1+\nu}\right) .
$$

The mapping is such that each edge of the parameter space corresponds to a quarter of circle, resulting to four singular points in the isogeometric mapping at the 


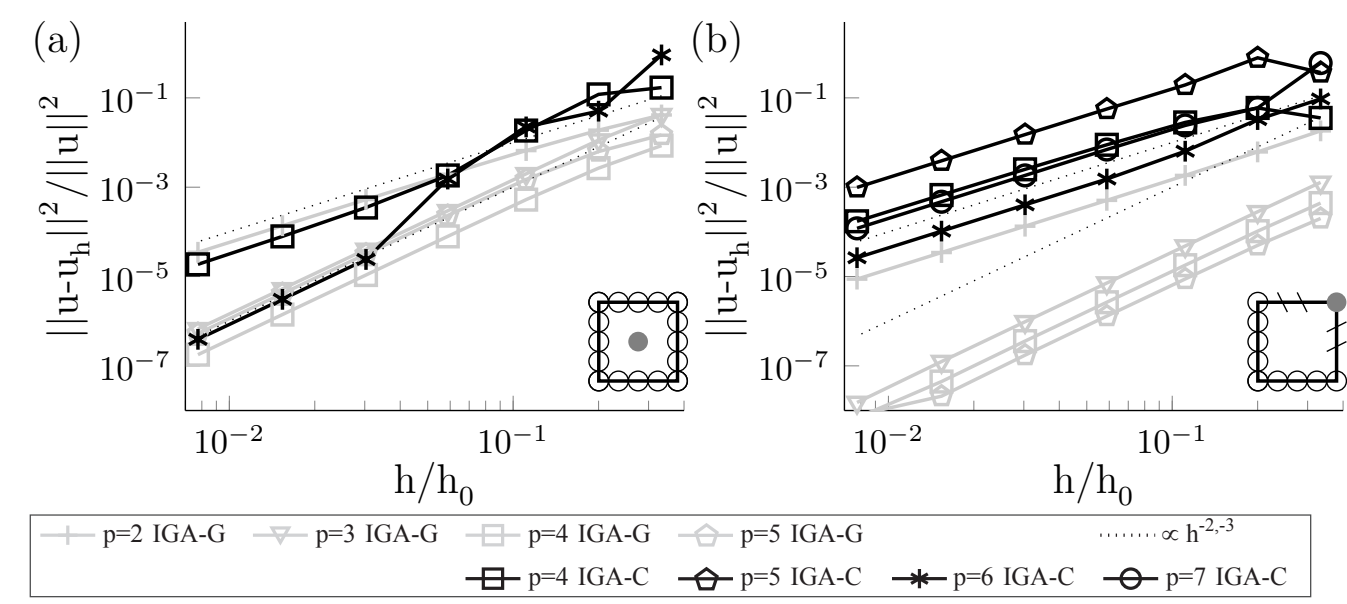

Figure 7: Relative $L^{2}$-error versus normalized mesh size $h / h_{0}$ for a SSSS plate with a point load in its middle (a), and a SSGG plate with a point load at the GG corner (b).

parametric space corners [58]. Convergence plots are shown in Figs. 8a-b and convergence orders are found in good agreement with estimated ones $(r=\infty)$, validating the collocation strategy, even in the presence of singular points, as already illustrated in [38].

\subsubsection{Annular plates}

Analytical solutions for an annular plate with a simply-supported outer edge and a free inner one are also available in [44]. With $R_{o}=1 \mathrm{~m}$ and $R_{i}=0.5 \mathrm{~m}$ denoting the outer and inner radius, respectively, the deflection for an annular plate under a constant linear force $\left(p_{3}^{\Gamma}=1 \mathrm{Nm}^{-1}\right)$ applied at the inner edge is given by

$$
\begin{aligned}
u_{3}(R)= & \frac{p_{3}^{\Gamma} R_{o}^{2} R_{i}}{4 D}\left(\left(1-\frac{R^{2}}{R_{o}^{2}}\right)\left(\frac{3+\nu}{2(1+\nu)}-\frac{R_{i}^{2}}{R_{o}^{2}-R_{i}^{2}} \ln \frac{R_{i}}{R_{o}}\right)\right. \\
& \left.+\frac{R^{2}}{R_{o}^{2}} \ln \frac{R}{R_{o}}+\frac{2 R_{i}^{2}}{R_{o}^{2}-R_{i}^{2}} \frac{1+\nu}{1-\nu} \ln \frac{R_{i}}{R_{o}} \ln \frac{R}{R_{o}}\right),
\end{aligned}
$$

whereas the deflection of the same plate under a distributed moment $c^{\Gamma}=1 \mathrm{Nm} / \mathrm{m}$ prescribed at the inner edge reads

$$
u_{3}(R)=\frac{c^{\Gamma} R_{i}^{2}}{D\left(R_{o}^{2}-R_{i}^{2}\right)}\left(\frac{R^{2}-R_{o}^{2}}{2(1+\nu)}+\frac{R_{o}^{2}}{1-\nu} \ln \frac{R}{R_{o}}\right) .
$$



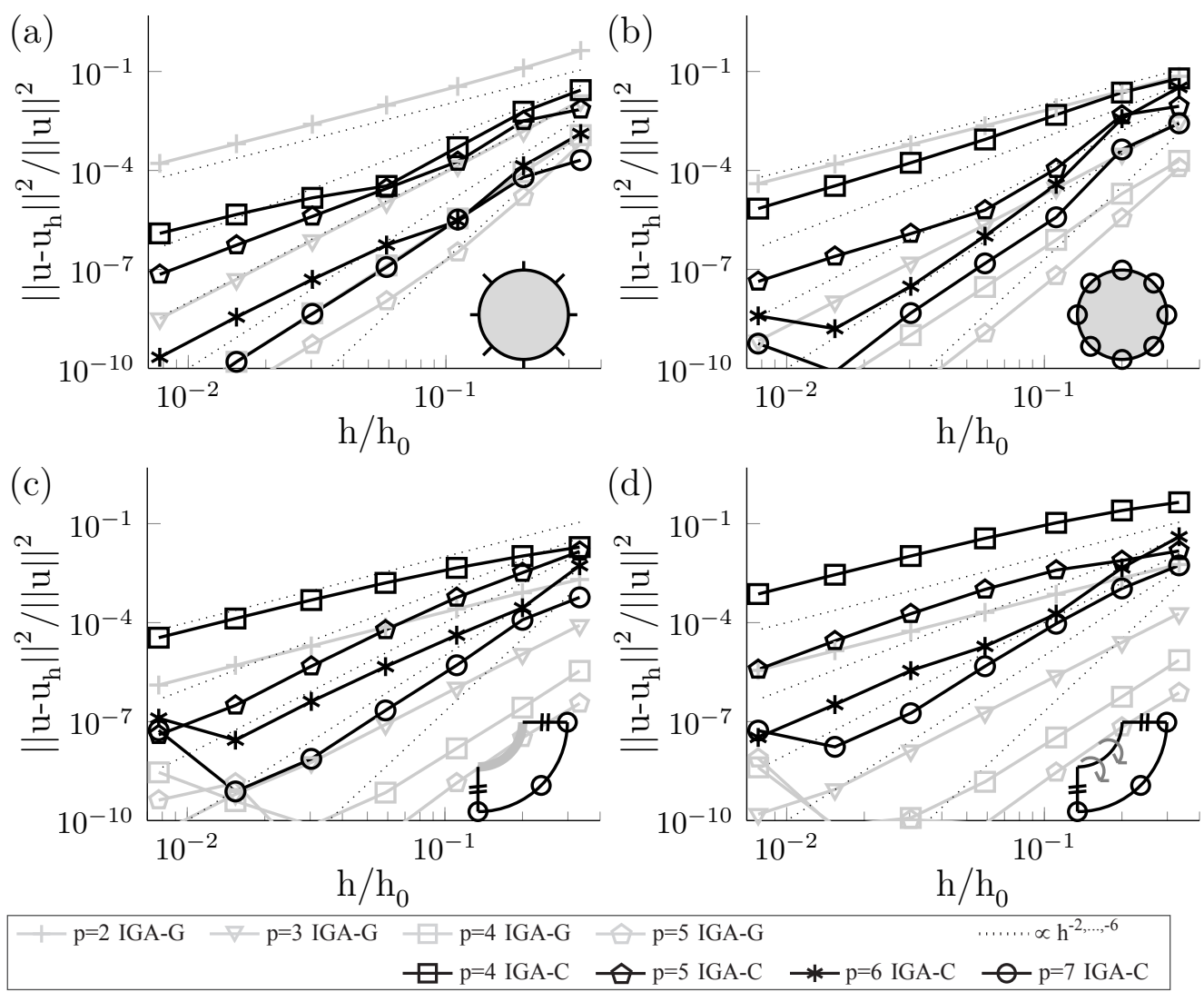

Figure 8: Relative $L^{2}$-error versus normalized mesh size $h / h_{0}$ for a uniformly loaded clamped (a) or simply-supported (b) circular plate, and for an annular plate simply supported at the outer edge, and free with a prescribed force (c) or moment (d) at the inner edge.

Taking advantage of symmetry, the model is restricted to one quarter of the annulus. Note that for the annular plate under a constant linear force, the solution has to be computed a posteriori, as detailed in Section 4.2.2. Convergence orders are found in good agreement with the a-priori estimated ones considering $r=\infty$ (Figs. 8c-d), except for high values of $p$, which can be the topic of future research.

\subsection{A non-uniformly curved shell}

In this example, the objective is to validate the formulation for non-uniformly curved geometries. The geometry is illustrated in Fig. 9 and it is constructed from the Coons patch of four $\mathcal{C}^{3}$-continuous B-spline curves, all based on the following $2 D$ B-spline: knot vector $\left\{\begin{array}{lllllllll}0 & 0 & 0 & 0 & 0 & 1 & 1 & 1 & 1\end{array}\right\}$, control points $\{\{0,0\}$ $\{L / 6,-L / 2\}\{L / 2,0\}\{5 L / 6, L / 2\}\{L, 0\}\}$, with $L=1 \mathrm{~m}$. All sides are 

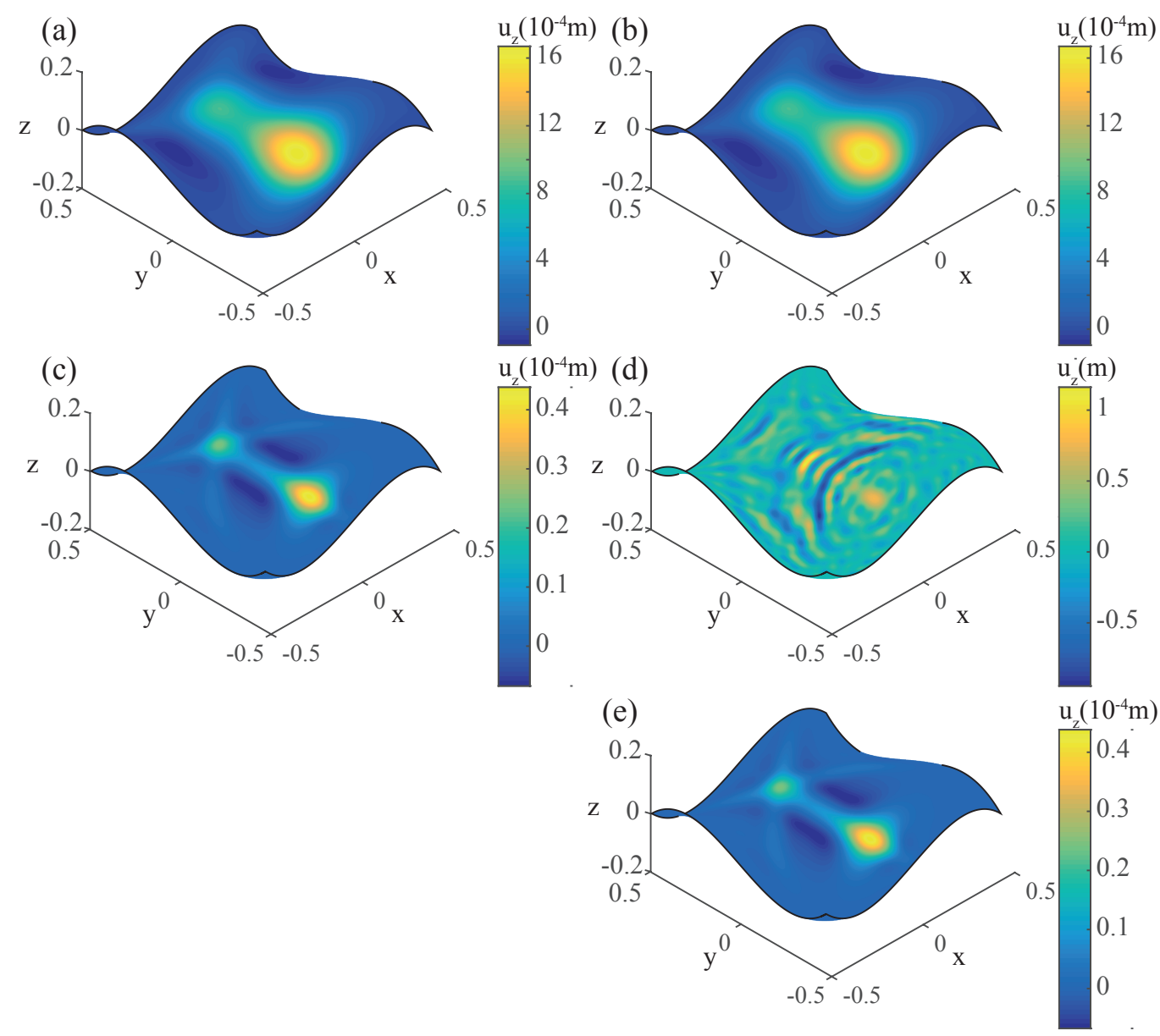

Figure 9: Component in the $z$ direction of the displacement field of the non-uniformly curved shell of thickness $5 \mathrm{~mm}(\mathrm{a}, \mathrm{b})$ and $0.1 \mathrm{~mm}(\mathrm{c}, \mathrm{d}, \mathrm{e})$, computed for IGA-G (a,c) and IGA-C (b,d,e) with $p=6, h_{0} / h=2^{6}+1$ (a-d) and $h_{0} / h=2^{8}+1$ (e).

clamped. The shell thickness is $t=5 \mathrm{~mm}$, the Young modulus is $E=200 \mathrm{GPa}$, and the Poisson ratio is $\nu=0.3$. The shell is loaded by a uniform pressure $p_{3}=1 \times 10^{5} \mathrm{Nm}^{-2}$.

The vertical displacement is reported for both IGA-G (Fig. 9a) and IGA-C (Fig. 9b) based on the same discretization, and they are found visually in agreement. Result validations are completed by a convergence analysis, and since no analytical solution is available for this problem, a reference is generated using IGA-G with a sufficiently fine mesh $\left(h_{0} / h=2^{7}+1\right)$ and shape functions of degree $p=7$. Convergence plots for the IGA-G and IGA-C methods provided in 


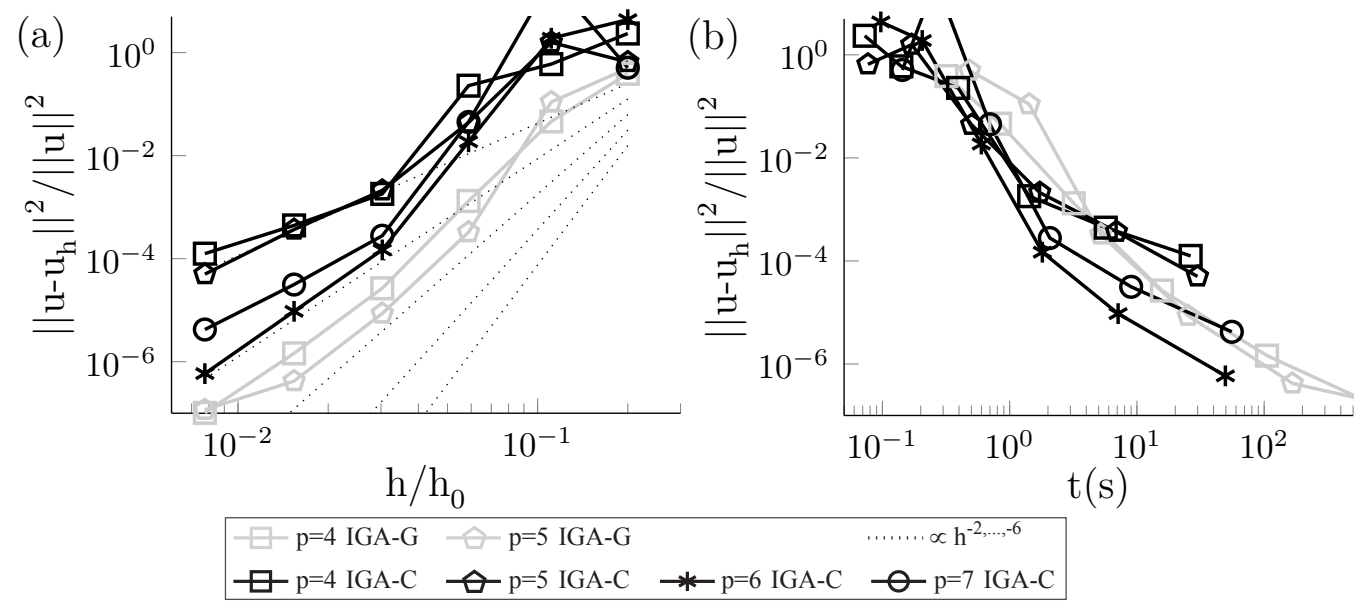

Figure 10: Relative $L^{2}$-error versus normalized mesh size $h / h_{0}$ (a) and versus computation time (b) of the non-uniformly curved shell of thickness $5 \mathrm{~mm}$. Results obtained with an Intel Core i7 $4720 H Q$ and taking advantage of sparse matrices.

Fig. 10a show that both methods converge, validating the implementation. Note that for IGA-C, the convergence is better for the even degrees than for the odd ones, but no proper explanation has been found yet.

Concerning the computation time, (Fig. 10b), the collocation method is about one order of magnitude faster than the Galerkin one for this application.

To complete this case study, the same problem with a smaller thickness $(t=$ $0.1 \mathrm{~mm}$ ) has been tested with $p=6$ and $h_{0} / h=2^{6}+1$. While the solution with IGA-G seems realistic (Fig. 9c), spurious oscillations (large peaks) are observed in the case of IGA-C (Fig. 9d). Note that if the mesh is refined ( $p=6$ and $h_{0} / h=2^{8}+1$ ), the correct solution is obtained (Fig. 9e), such that a possible explanation could be membrane locking, as observed in [59].

\subsection{Scordelis-Lo roof}

In this example, the Scordelis-Lo roof $[10,16,17,19,20]$ is investigated, demonstrating the application of the collocation method to a curved geometry with Neumann boundary conditions. The Scordelis-Lo roof is an $80^{\circ}$ cylinder section of length $L=50 \mathrm{~m}$ and radius $R=25 \mathrm{~m}$, loaded by a uniform body force of $-90 \mathrm{Nm}^{-2}$ along the vertical direction ( $z$ axis, see Fig. 11). The thickness is $t=0.25 \mathrm{~m}$, the Young modulus is $E=432 \mathrm{MPa}$, and the Poisson ratio is $\nu=0$.

The vertical deflection field for IGA-C is reported in Fig. 11. Contrary to previous works on collocation where Neumann boundary conditions are investi- 


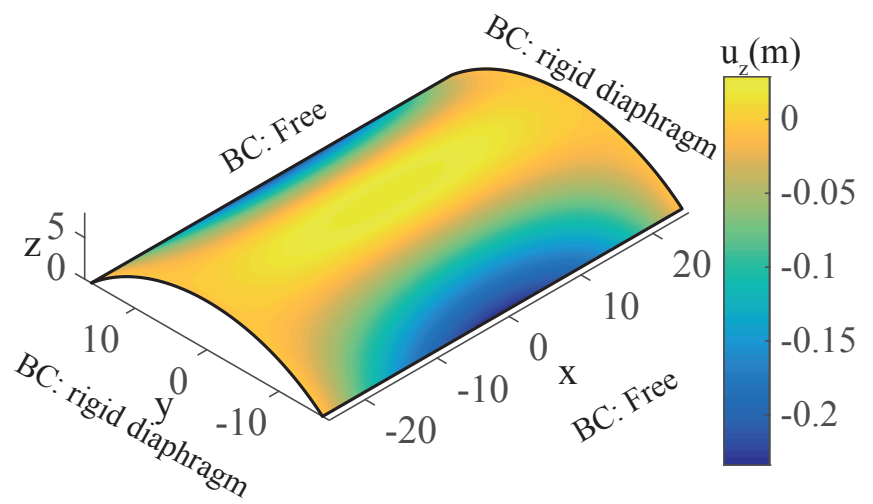

Figure 11: Component in the $z$ direction of the displacement field of the Scordelis-Lo roof, computed with the IGA-C with $p=6$ and $h_{0} / h=2^{6}+1$.

gated, no oscillation on the displacement field is observed, avoiding the use of the hybrid collocation-Galerkin method [23, 24]. The convergence curves of the relative displacement error at the mid-span of the free edge (using as reference $-0.300592457 \mathrm{~m}$ [20]) are reported in Fig. 12a, and both discretization methods are found to converge. Moreover, while the error in terms of the computation time seems to be optimal with $p=4$ for IGA-G, for IGA-C, this order is much higher and is around $p=10$ (Fig. 12b). However, contrary to the previous example IGA-C is no more attractive in terms of computation cost, except if really accurate results are required.

\subsection{The pinched cylinder}

In this example, the pinched cylinder $[10,12,16,17]$ is investigated, demonstrating the application of the collocation method to a curved geometry with point loads. The pinched cylinder is supported by rigid diaphragms at the extremities and is loaded by opposite radial concentrated forces of magnitude $P=-1 \mathrm{~N}$ at its mid-span. The cylinder has a section of length $L=600 \mathrm{~mm}$ and radius $R=$ $300 \mathrm{~mm}$, the thickness is $t=3 \mathrm{~mm}$, the Young modulus is $E=3000000 \mathrm{Nmm}^{-2}$, and the Poisson ratio is $\nu=0.3$.

To illustrate the computation cost differences between a concentrated force applied inside the span and at its corner, the symmetries of the problem are used to generate two models. One made of one half of the full cylinder with the point force located at its mid-span, and one modeled by an eighth of the full cylinder with the force at one of its corner (see Fig. 13). Note that for the "half" model, the half circle curve is generated from a cubic single NURBS patch defined by the 


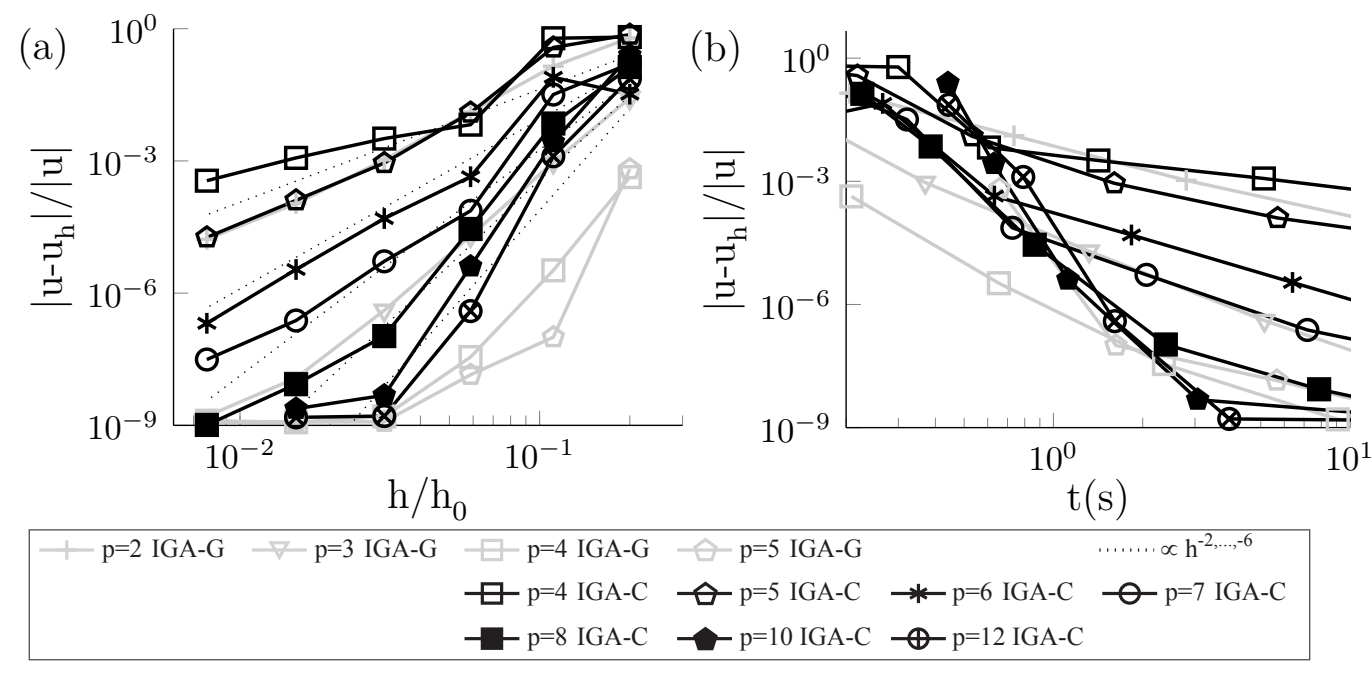

Figure 12: Relative error at the mid-span versus normalized mesh size $h / h_{0}$ (a) and versus computation time (b) of the Scordelis-Lo roof. Results obtained with an Intel Core i7 $4720 H Q$ and taking advantage of sparse matrices.
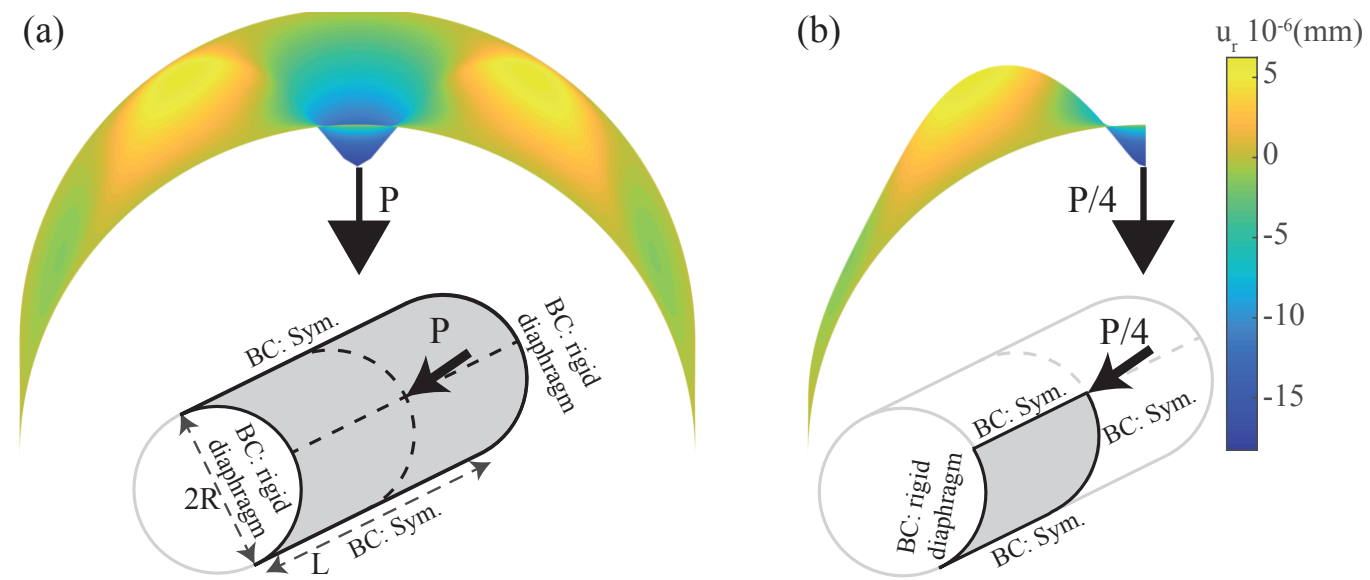

Figure 13: Radial displacement field scaled by a factor $5 \times 10^{6}$ of the pinched cylinder modeling only its half (a) and its eighth (b), computed with the IGA-C with $p=6$ and $h_{0} / h=2^{6}+1$.

control points $\{\{0,-R\},\{2 R,-R\},\{2 R, R\},\{0, R\}\}$ and the weights $\{1,1 / 3$, $1 / 3,1\}$.

The convergence curves of the relative displacement error at the point load are reported in Figs. 14a and 14c for the half and the eighth models, respectively. As a reference, $u_{r}=1.8248 \times 10^{-5} \mathrm{~mm}$ has been initially used in the literature 

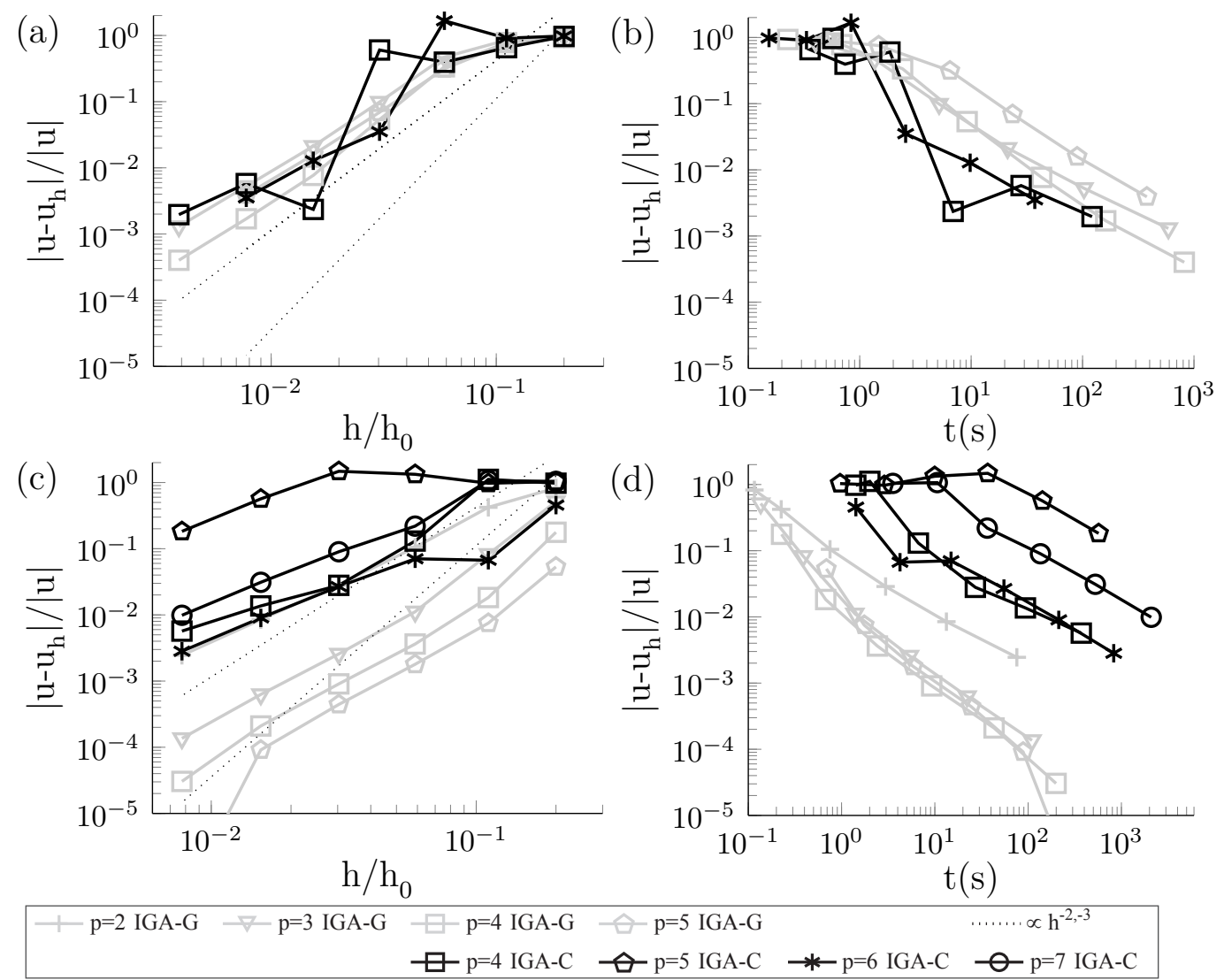

Figure 14: Relative error at the point load versus normalized mesh size $h / h_{0}$ (a,c) and versus computation time $(b, d)$ of the half $(a, b)$ and the eighth $(c, d)$ pinched cylinder. Results obtained with an Intel Core $i 74720 H Q$ and taking advantage of sparse matrices. Note that for (a-b), odd NURBS degrees are not considered for IGA-C, since it has been assumed in Section 4.2.2 that a collocation point has to be present at the point force location, which is not the case with CSP and a regular mesh.

[10], but it is shown in [12] that a better estimation is $u_{r}=1.82715797 \times 10^{-5}$ $\mathrm{mm}$. However, as stressed in [12], this last value results from an infinite series that converge in theory but not numerically due to the Gibbs effect [60]. For this reason, we use here a value directly computed with IGA-G, $p=7$ and $h_{0} / h=$ $2^{7}+1$ which is $u_{r}=1.828140 \times 10^{-5} \mathrm{~mm}$.

In Fig. 14, all the curves are found to converge but better results are obtained when the load is applied directly at the corner, since at that point, the mapping is interpolatory. 
For the computation time, while the use of IGA-C is competitive with respect to IGA-G when the load is located in the middle of the mid-span (Fig. 14b), IGA$\mathrm{C}$ turns out to be more than one order of magnitude slower than IGA-G when the load is at the corner. This is explained by the strategy used to solve this problem which requires the integration of the fourth-order problem (Eq. (74)), combining the disadvantage of both the Galerkin and the collocation methods. More efficient solutions could be the topic of further research.

\section{Conclusions}

We proposed the isogeometric collocation of Kirchhoff-Love shells under the assumption of small deformations. This method is valid for all possible combinations of adjacent edge boundary conditions, after the implementation of priority and averaging rules based on the boundary types. Greville and alternative superconvergent points are used for NURBS basis of even and odd degrees, respectively. The convergence orders in the $L^{2}$-error norm are found to be in agreement with a-priori error estimates.

Despite a higher degree of complexity of the collocation method, computational gains may prove very attractive for some specific Kirchhoff-Love shell problems, with respect to the classical Galerkin approach and its costly numerical integration steps. However, we believe that taking advantage of the most recent improvements in reduced quadrature rules would make the Galerkin method computationally more attractive [61-65].

\section{Acknowledgments}

The Research Fund KU Leuven is gratefully acknowledged for its support. This research was also partially supported by Flanders Make, the strategic research center for the manufacturing industry. The research of F. Maurin is funded by an Experienced Researcher grant within the European ANTARES Project, under the FP7 Marie Curie Programme (GA606817). The research of L. Coox is funded by a grant from the IWT Flanders.

\section{References}

[1] T. Hughes, J. Cottrell, and Y. Bazilevs, "Isogeometric analysis: CAD, finite elements, NURBS, exact geometry and mesh refinement," Computer Methods in Applied Mechanics and Engineering, vol. 194, no. 39, pp. 4135-4195, 2005. 
[2] J. A. Cottrell, T. J. R. Hughes, and Y. Bazilevs, Isogeometric Analysis Toward Intergration of CAD and FEA. John Wiley \& Sons, 2009.

[3] F. Auricchio, L. B. da Veiga, A. Buffa, C. Lovadina, A. Reali, and G. Sangalli, "A fully "locking-free" isogeometric approach for plane linear elasticity problems: A stream function formulation," Computer Methods in Applied Mechanics and Engineering, vol. 197, pp. 160-172, dec 2007.

[4] H. Gómez, V. M. Calo, Y. Bazilevs, and T. J. R. Hughes, "Isogeometric analysis of the CahnHilliard phase-field model," Computer Methods in Applied Mechanics and Engineering, vol. 197, pp. 4333-4352, sep 2008.

[5] A. Bartezzaghi, L. Dedè, and A. Quarteroni, "Isogeometric Analysis of high order Partial Differential Equations on surfaces," Computer Methods in Applied Mechanics and Engineering, vol. 295, pp. 446-469, oct 2015.

[6] A. Tagliabue, L. Dedè, and A. Quarteroni, "Isogeometric Analysis and error estimates for high order partial differential equations in fluid dynamics," Computers \& Fluids, vol. 102, pp. 277-303, oct 2014.

[7] S. B. Raknes, X. Deng, Y. Bazilevs, D. J. Benson, K. M. Mathisen, and T. Kvamsdal, "Isogeometric rotation-free bending-stabilized cables: Statics, dynamics, bending strips and coupling with shells," Computer Methods in Applied Mechanics and Engineering, vol. 263, pp. 127-143, aug 2013.

[8] F. Maurin, L. Dedè, and A. Spadoni, "Isogeometric rotation-free analysis of planar extensible-elastica for static and dynamic applications," Nonlinear Dynamics, vol. 81, pp. 77-96, mar 2015.

[9] L. Greco and M. Cuomo, "B-Spline interpolation of Kirchhoff-Love space rods," Computer Methods in Applied Mechanics and Engineering, vol. 256, no. 0, pp. 251-269, 2013.

[10] J. Kiendl, K. U. Bletzinger, J. Linhard, and R. Wüchner, "Isogeometric shell analysis with Kirchhoff-Love elements," Computer Methods in Applied Mechanics and Engineering, vol. 198, no. 49-52, pp. 3902-3914, 2009.

[11] J. Kiendl, M. C. Hsu, M. C. H. Wu, and A. Reali, "Isogeometric KirchhoffLove shell formulations for general hyperelastic materials," Computer Methods in Applied Mechanics and Engineering, vol. 291, pp. 280-303, 2015. 
[12] T. X. Duong, F. Roohbakhshan, and R. A. Sauer, "A new rotation-free isogeometric thin shell formulation and a corresponding continuity constraint for patch boundaries," Computer Methods in Applied Mechanics and Engineering, vol. 316, pp. 43-83, 2017.

[13] N. Nguyen-Thanh, N. Valizadeh, M. N. Nguyen, H. Nguyen-Xuan, X. Zhuang, P. Areias, G. Zi, Y. Bazilevs, L. De Lorenzis, and T. Rabczuk, "An extended isogeometric thin shell analysis based on Kirchhoff-Love theory," Computer Methods in Applied Mechanics and Engineering, vol. 284, pp. 265-291, feb 2015.

[14] X. Deng, A. Korobenko, J. Yan, and Y. Bazilevs, "Isogeometric analysis of continuum damage in rotation-free composite shells," Computer Methods in Applied Mechanics and Engineering, vol. 284, pp. 349-372, 2015.

[15] A. B. Tepole, H. Kabaria, K.-U. Bletzinger, and E. Kuhl, "Isogeometric KirchhoffLove shell formulations for biological membranes," Computer Methods in Applied Mechanics and Engineering, vol. 293, pp. 328-347, 2015.

[16] J. Kiendl, Y. Bazilevs, M. C. Hsu, R. Wüchner, and K. U. Bletzinger, "The bending strip method for isogeometric analysis of Kirchhoff-Love shell structures comprised of multiple patches," Computer Methods in Applied Mechanics and Engineering, vol. 199, no. 37-40, pp. 2403-2416, 2010.

[17] Y. Guo and M. Ruess, "Nitsche's method for a coupling of isogeometric thin shells and blended shell structures," Computer Methods in Applied Mechanics and Engineering, vol. 284, pp. 881-905, 2015.

[18] F. Maurin, L. Coox, F. Greco, E. Deckers, C. Claeys, and W. Desmet, "Bloch theorem for isogeometric analysis of periodic problems governed by highorder partial differential equations," Computer Methods in Applied Mechanics and Engineering, vol. 311, pp. 743-763, 2016.

[19] A. Goyal and B. Simeon, "On penalty-free formulations for multipatch isogeometric KirchhoffLove shells," Mathematics and Computers in Simulation, 2016.

[20] L. Coox, F. Maurin, F. Greco, E. Deckers, D. Vandepitte, and W. Desmet, "A flexible approach for coupling NURBS patches in rotationless isogeometric 
analysis of KirchhoffLove shells," Computer Methods in Applied Mechanics and Engineering, vol. 325, pp. 505-531, oct 2017.

[21] F. Auricchio, L. B. Da Veiga, T. J. R. Hughes, A. Reali, and G. Sangalli, "Isogeometric Collocation Methods," Mathematical Models and Methods in Applied Sciences, vol. 20, pp. 2075-2107, nov 2010.

[22] D. Schillinger, J. A. Evans, A. Reali, M. A. Scott, and T. J. R. Hughes, "Isogeometric collocation: Cost comparison with Galerkin methods and extension to adaptive hierarchical NURBS discretizations," Computer Methods in Applied Mechanics and Engineering, vol. 267, pp. 170-232, dec 2013.

[23] L. De Lorenzis, J. Evans, T. Hughes, and A. Reali, "Isogeometric collocation: Neumann boundary conditions and contact," Computer Methods in Applied Mechanics and Engineering, vol. 284, pp. 21-54, 2015.

[24] R. Kruse, N. Nguyen-Thanh, L. De Lorenzis, and T. Hughes, "Isogeometric collocation for large deformation elasticity and frictional contact problems," Computer Methods in Applied Mechanics and Engineering, vol. 296, pp. 73$112,2015$.

[25] F. Auricchio, L. Beirão da Veiga, T. J. R. Hughes, A. Reali, and G. Sangalli, "Isogeometric collocation for elastostatics and explicit dynamics," Computer Methods in Applied Mechanics and Engineering, vol. 249-252, pp. 2-14, dec 2012.

[26] H. Gomez, A. Reali, and G. Sangalli, "Accurate, efficient, and (iso)geometrically flexible collocation methods for phase-field models," Journal of Computational Physics, vol. 262, pp. 153-171, 2014.

[27] D. Schillinger, M. J. Borden, and H. K. Stolarski, "Isogeometric collocation for phase-field fracture models," Computer Methods in Applied Mechanics and Engineering, vol. 284, pp. 583-610, 2015.

[28] O. Weeger, B. Narayanan, L. De Lorenzis, J. Kiendl, and M. L. Dunn, "An isogeometric collocation method for frictionless contact of Cosserat rods," Computer Methods in Applied Mechanics and Engineering, vol. 321, pp. 361-382, 2017.

[29] L. Beirão da Veiga, C. Lovadina, and A. Reali, "Avoiding shear locking for the Timoshenko beam problem via isogeometric collocation methods," 
Computer Methods in Applied Mechanics and Engineering, vol. 241-244, pp. 38-51, oct 2012.

[30] F. Auricchio, L. Beirão da Veiga, J. Kiendl, C. Lovadina, and A. Reali, "Locking-free isogeometric collocation methods for spatial Timoshenko rods," Computer Methods in Applied Mechanics and Engineering, vol. 263, pp. 113-126, 2013.

[31] J. Kiendl, F. Auricchio, T. J. R. Hughes, and A. Reali, "Single-variable formulations and isogeometric discretizations for shear deformable beams," Computer Methods in Applied Mechanics and Engineering, vol. 284, pp. 988-1004, 2015.

[32] O. Weeger, S.-K. Yeung, and M. L. Dunn, "Isogeometric collocation methods for Cosserat rods and rod structures," Computer Methods in Applied Mechanics and Engineering, 2016.

[33] J. Kiendl, F. Auricchio, and A. Reali, "A displacement-free formulation for the Timoshenko beam problem and a corresponding isogeometric collocation approach," Meccanica, pp. 1-11, aug 2017.

[34] G. Balduzzi, S. Morganti, F. Auricchio, and A. Reali, "Non-prismatic Timoshenko-like beam model: Numerical solution via isogeometric collocation," Computers \& Mathematics with Applications, 2017.

[35] J. Kiendl, F. Auricchio, L. Beirão da Veiga, C. Lovadina, and A. Reali, "Isogeometric collocation methods for the Reissner-Mindlin plate problem," Computer Methods in Applied Mechanics and Engineering, vol. 284, pp. 489-507, 2015.

[36] G. Pavan and K. Nanjunda Rao, "Bending analysis of laminated composite plates using isogeometric collocation method," Composite Structures, vol. 176, pp. 715-728, sep 2017.

[37] J. Kiendl, E. Marino, and L. De Lorenzis, "Isogeometric collocation for the ReissnerMindlin shell problem," Computer Methods in Applied Mechanics and Engineering, vol. 325, pp. 645-665, 2017.

[38] A. Reali and H. Gomez, "An isogeometric collocation approach for Bernoulli-Euler beams and Kirchhoff plates," Computer Methods in Applied Mechanics and Engineering, vol. 284, pp. 623-636, 2015. 
[39] H. Casquero, L. Liu, Y. Zhang, A. Reali, and H. Gomez, "Isogeometric collocation using analysis-suitable T-splines of arbitrary degree," Computer Methods in Applied Mechanics and Engineering, vol. 301, pp. 164-186, 2016.

[40] C. Anitescu, Y. Jia, Y. J. Zhang, and T. Rabczuk, "An isogeometric collocation method using superconvergent points," Computer Methods in Applied Mechanics and Engineering, vol. 284, pp. 1073-1097, 2015.

[41] H. Gomez and L. De Lorenzis, "The variational collocation method," Computer Methods in Applied Mechanics and Engineering, vol. 309, pp. 152181, 2016.

[42] M. Montardini, G. Sangalli, and L. Tamellini, "Optimal-order isogeometric collocation at Galerkin superconvergent points," Computer Methods in Applied Mechanics and Engineering, vol. 316, pp. 741-757, 2017.

[43] Y. Basar and W. B. Kratzig, Mechanik der Flachentragwerke : Theorie, Berechnungsmethoden, Anwendungsbeispiele. Vieweg, 1985.

[44] A. C. Ugural, Stresses in Beams, Plates, and Shells, Third Edition. CRC Press, 2009.

[45] L. Piegl and W. Tiller, The NURBS Book. Springer Science \& Business Media, 1996.

[46] M. Spink, D. Claxton, C. de Falco, and R. Vazquez, "The NURBS toolbox," 2017.

[47] C. de Falco, A. Reali, and R. Vázquez, "GeoPDEs: A research tool for Isogeometric Analysis of PDEs," Advances in Engineering Software, vol. 42, pp. 1020-1034, dec 2011.

[48] A. Reali and T. J. R. Hughes, "An Introduction to Isogeometric Collocation Methods," pp. 173-204, Springer Vienna, 2015.

[49] H. Melzer and R. Rannacher, "Spannungskonzentrationen in Eckpunkten der vertikal belasteten Kirchhoffschen Platte," Bauingenieur, 1979.

[50] H. Blum, R. Rannacher, and R. Leis, "On the boundary value problem of the biharmonic operator on domains with angular corners," Mathematical Methods in the Applied Sciences, vol. 2, no. 4, pp. 556-581, 1980. 
[51] L. Beirão da Veiga, J. Niiranen, and R. Stenberg, "A family of C0 finite elements for Kirchhoff plates II: Numerical results," Computer Methods in Applied Mechanics and Engineering, vol. 197, pp. 1850-1864, apr 2008.

[52] M. Batista, "Uniformly Loaded Rectangular Thin Plates with Symmetrical Boundary Conditions," arXiv preprint, jan 2010.

[53] C. Lim, S. Cui, and W. Yao, "On new symplectic elasticity approach for exact bending solutions of rectangular thin plates with two opposite sides simply supported," International Journal of Solids and Structures, vol. 44, no. 16, pp. 5396-5411, 2007.

[54] Y. Liu and R. Li, "Accurate bending analysis of rectangular plates with two adjacent edges free and the others clamped or simply supported based on new symplectic approach," Applied Mathematical Modelling, vol. 34, no. 4, pp. 856-865, 2010.

[55] M. Batista, "New analytical solution for bending problem of uniformly loaded rectangular plate supported on corner points," The IES Journal Part A: Civil \& Structural Engineering, vol. 3, pp. 75-84, may 2010.

[56] S. Timoshenko and S. Woinowsky-Krieger, Theory of plates and shells. McGraw-Hill, 1959.

[57] L. H. Nguyen and D. Schillinger, "A collocated isogeometric finite element method based on GaussLobatto Lagrange extraction of splines," Computer Methods in Applied Mechanics and Engineering, vol. 316, pp. 720-740, 2017.

[58] A.-V. Vuong, C. Heinrich, and B. Simeon, "ISOGAT: A 2D tutorial MATLAB code for Isogeometric Analysis," Computer Aided Geometric Design, vol. 27, pp. 644-655, nov 2010.

[59] D. Schillinger, J. A. Evans, F. Frischmann, R. R. Hiemstra, M.-C. Hsu, and T. J. R. Hughes, "A collocated C0 finite element method: Reduced quadrature perspective, cost comparison with standard finite elements, and explicit structural dynamics," International Journal for Numerical Methods in Engineering, vol. 102, no. 3-4, pp. 576-631, 2015.

[60] A. J. Jerri, The Gibbs Phenomenon in Fourier Analysis, Splines and Wavelet Approximations. Boston, MA: Springer US, 1998. 
[61] A. Mantzaflaris and B. Jüttler, "Integration by interpolation and look-up for Galerkin-based isogeometric analysis," Computer Methods in Applied Mechanics and Engineering, vol. 284, pp. 373-400, feb 2015.

[62] P. Antolin, A. Buffa, F. Calabrò, M. Martinelli, and G. Sangalli, "Efficient matrix computation for tensor-product isogeometric analysis: The use of sum factorization," Computer Methods in Applied Mechanics and Engineering, vol. 285, pp. 817-828, mar 2015.

[63] R. R. Hiemstra, F. Calabrò, D. Schillinger, and T. J. R. Hughes, "Optimal and reduced quadrature rules for tensor product and hierarchically refined splines in isogeometric analysis," Computer Methods in Applied Mechanics and Engineering, 2016.

[64] M. Barto and V. M. Calo, "Optimal quadrature rules for odd-degree spline spaces and their application to tensor-product-based isogeometric analysis," Computer Methods in Applied Mechanics and Engineering, vol. 305, pp. 217-240, jun 2016.

[65] K. A. Johannessen, "Optimal quadrature for univariate and tensor product splines," Computer Methods in Applied Mechanics and Engineering, vol. 316, pp. 84-99, 2017. 